\title{
Information Flow between Global Equities and Cryptocurrencies: A VMD-Based Entropy Evaluating Shocks from COVID-19 Pandemic
}

\author{
Emmanuel Asafo-Adjei (D, Peterson Owusu Junior (D), and Anokye M. Adam (i) \\ Department of Finance, School of Business, University of Cape Coast, Cape Coast, Ghana \\ Correspondence should be addressed to Emmanuel Asafo-Adjei; eaadjei12998@gmail.com
}

Received 17 August 2021; Revised 15 September 2021; Accepted 20 September 2021; Published 21 October 2021

Academic Editor: Paulo Jorge Silveira Ferreira

Copyright ( $\odot 2021$ Emmanuel Asafo-Adjei et al. This is an open access article distributed under the Creative Commons Attribution License, which permits unrestricted use, distribution, and reproduction in any medium, provided the original work is properly cited.

\begin{abstract}
The world has witnessed the adverse impact of the COVID-19 pandemic. Accordingly, it is expected that information transmission between equities and digital assets has been altered due to the hostile impact of the pandemic outbreak on financial markets. As a result, the ensuing perverse risk among markets is presumed to rise during severe uncertainties occasioned by the COVID-19 pandemic. The impetus of this study is to examine the degree of asymmetry and nonlinear directional causality between global equities and cryptocurrencies in the frequency domain. Hence, we employ both the variational mode decomposition (VMD) and the Rényi effective transfer entropy techniques. Analyses of the study are presented for three sample periods; these are the full sample period, the pre-COVID-19 period, and the COVID-19 pandemic period. We gauge a mixture of asymmetric and nonlinear bidirectional and unidirectional causality between global equities and cryptocurrencies for the sample periods. However, the COVID-19 pandemic period appears to be driving the estimates for the full sample period, which indicates a negative flow. Thus, the direction and significance of the information flow between the markets for the full sample correspond to the one observed during the COVID-19 pandemic period. We, consequently, establish a significant directional, dynamical, and scale-dependent information flow between global equities and cryptocurrencies. Notwithstanding, throughout the study samples, we mainly find a negative significant information flow from global equities to cryptocurrencies. We detect that most cryptocurrencies exhibit similar behaviour of information flow to global equities for each of the sample periods. The outcome provides pertinent signals to investors with diverse investment horizons who would want to diversify, hedge, or employ cryptocurrencies as a safe haven for global equities during uncertainties, specifically the COVID-19 pandemic.
\end{abstract}

\section{Introduction}

The advent of the COVID-19 pandemic has induced many empirical studies on economies, including its impact on mental health [1], food prices [2], interdependencies between COVID-19 and exchange rates in Wuhan [3], COVID-19 and Bitcoin [4], the nexus between equities and cryptocurrencies [5], crude oil and agricultural futures [6], major world markets [7], and mutual information between markets [8], among others. It can be analysed from these studies that the impact of the COVID-19 has disrupted most economic activities including financial markets of which cryptocurrencies and global equities are no exception.
The world all over has witnessed the substantial expansion of digital currencies. The seminar paper of Chaum [9] indicates that cryptocurrencies rely on cryptographic proofs for transaction confirmation and were developed in 1983 [10]. Nakamoto [11] published the Bitcoin project and asserted that anonymity, lack of control from a central authority, and protection from double-spending are all features of cryptocurrencies. The popularity of cryptocurrencies increased at the advent of the European sovereign debt crisis of 2010-2013 [12]. The market size of cryptocurrencies has not stopped growing; in January 2018, market capitalization peaked at USD 800 billion as compared to a little above USD 10 billion in 2013 [12, 13]. Notwithstanding, 
in September 2018, cryptocurrency markets faced extreme price instability which resulted in an $80 \%$ loss of their values [14]. Cryptocurrencies are susceptible to speculative bubbles since it is characterised by anonymity [15]. According to Yarovaya et al. [16], speculative bubbles in cryptocurrency marketplaces might spread contagion and compromise financial stability. As a result, a study that mixes cryptocurrencies with other assets, such as equities, is critical for investment decisions since it establishes information transmission patterns [15]. Although other financial assets, especially stocks, are influenced by several factors [17], the distinct feature of stocks may welcome diversification benefits from cryptocurrencies [15, 18].

The arbitrage pricing theory, projected by Ross [19], asserts that stock returns are theorised to be influenced by several factors and had been confirmed by extant literature $[20,21]$. Furthermore, prior literature offers an indication that the stock market returns are influenced by four main factors [12]. These are the fundamental factors, earning base [22]; the technical factors that encompass diverse macroeconomic factors [23]; market sentiment factors, animal spirits of investors and environmental context [24]; and market anomalies [25]. This study contributes to prior literature by incorporating the flow of information between global equities and cryptocurrencies, due to their increased market integration [26]. The degree of integration between these markets necessitates the assessment of diversification, hedge, and safe haven benefits [15].

The financial market integration theory has made it possible for the financial markets in an economy to become more closely related with those in other economies around the world [27-29]. The advancement of market integration is altered by institutional and behavioural factors [30]. To begin with, integration is a result of institutional, economic, and political reforms. Integration is dependent on global investors' capacity to access domestic securities as well as local investors' ability to access overseas investment possibilities, legally or otherwise $[28,30]$. Access to global international investment options, whether by legal means, home-grown diversification, or illegal means, enhances the exposure of domestic assets to the global factor and so raises the level of national stock market integration $[29,30]$. For instance, a study by Owusu Junior et al. [31] revealed the connectedness of most financial markets in emerging market economies. Risk preference, relative optimism, and information perception are all behavioural characteristics that might influence the willingness to invest overseas, and hence, market integration is amplified [30]. These dynamics are not far from the operations of cryptocurrencies and global equity markets by market participants. As a result, investments in cryptocurrencies and equities have become increasingly important to the global investment community in recent years. This is so, for a variety of reasons, including expectations of increased domination across the globe and extreme changes in capital flows between markets [31]. Consequently, improvement in the flow of capital and susceptibility of prices and returns to traded financial assets in diverse markets balance.
Despite the intense expansion of digital assets, there are limited studies that devote interest to finding the flow of information with equity returns, specifically global equities. Improvement in the globalisation of business enterprises and the integration of capital markets are modifying the equity investment environment. Foreign countries were once thought to be a diversifying alternative to an investor's equity market, so equity investing used to be primarily based on a series of country-defined markets [32]. This suggests that the domicile of a corporation is an insufficient indication of its operations. Its revenues, underlying active capital stock, and financing have all shifted to a more global scale [32]. We, therefore, conceptualise the global equities to mean the risk capital available for deployment internationally within various investment avenues based on the investors' level of risk aversion. For this study, global equities are represented by global market indices such as the Global Real Estate Investment Trust Equities (GREITs), NASDAQ Global Market Composite Index (GMI), Eurozone Index (EUROZI), Europe Index (EUROPI), Emerging Markets Index (EMI), and Developed Markets Index (DMI). We employ these equity indices to examine information flow with cryptocurrencies without considering country-specific stocks to carefully illustrate the competitive dynamics within these markets. As a result, country-specific stock market indices may not provide adequate information to examining information flow with cryptocurrencies which are most likely to transmit speculative bubbles to most financial assets and weaken their stability [16]. We do this to reiterate the competitive market hypothesis $(\mathrm{CMH})$ of Owusu Junior et al. [33]. The hypothesis implies that, in part, the intensity of information flows and spillover between markets of the same and differing asset classes are intensified by rational, albeit irrational investors' relentless search of competing rewards and risks to satisfy the portfolio goals. We, therefore, assess the degree of competitiveness within these markets to either act as a substitute or complement for each other.

Cryptocurrencies and equities are considered by extant literature to be assets through which investors can channel their investments [18] despite their underdeveloped relationships. Although the cryptocurrency economy is primarily different from the equity markets, both of the markets function in the same way. For instance, their prices are determined by demand and are valued in fiat currencies. Investors of equities may hedge against volatility in the equities market using cryptocurrencies [34]. This is due to the fear of volatility in demand for equities and loss of currency value, which may plummet asset prices, occasioned by macroeconomic conditions. This is so because cryptocurrencies values are hardly influenced by macroeconomic conditions, especially specific to a given country [35]. For example, fluctuations in exchange rates, interest rates, and inflation may not have a clear connection with the values of cryptocurrencies, because the cryptocurrencies markets are not regulated by a central authority [36]. On the other hand, the equity parity theory addresses the extent to which fluctuations in international investment holdings, such as cryptocurrencies, correspond to fluctuations in stocks. That 
is as individuals invest in cryptocurrencies as an international holding, domestic equity holdings are exposed to adverse exchange rates shocks.

Nonetheless, the creation of new financial or digital assets may stimulate investors to channel part or all of their investments between markets based on the level of their risk aversion. Building from Markowitz [37] portfolio theory, there is an alternative asset (market) within which investors may channel part or all of their investments at their free will, but with the quest of either maximising expected returns or minimising risk on the portfolio. This investment mechanism also establishes the flow of information between markets due to the competitive nature of the markets, as indicated by the information flow theory. For this study, since cryptocurrencies and equities are widely opened to global investors, we hypothesise a significant flow of information between global equities and cryptocurrencies. This would serve as either competitive alternate markets or complementary markets for global investors with diverse investment horizons [38], coupled with the nonstationarity, asymmetry, and nonlinearity of financial time series.

In nature, many unstable signals typically contain essential details [39]. Signal decomposition is a useful technique for dealing with nonstationary signals since it can break down a multifaceted signal into many standard simple modes that can be simply analysed in the time and/or frequency domain. Owing to the ubiquitous behaviour of unstable signals, financial time series are surrounded by noise and experience rapid oscillations of which cryptocurrencies and equities are no exception $[5,10]$. The intricacy of the price generation process catechises the validity of the natural rule of scale-invariance of a self-similar process to support the heterogeneous market hypothesis [38]. Market players are viewed as heterogeneous by the heterogeneous market hypothesis (HMH), with a wide range of information, purposes, and investment horizons [10,33]. Market participants respond to information at different times, resulting in very noisy market data. Consequently, cryptocurrency and global stock price series exhibit nonlinearity and nonstationarity. Information that misrepresents unpretentious core patterns is referred to as noise. Small price corrections in the market, as well as price variations that distort the broader trend, are examples of noise in the financial markets. This suggests that market noise might make it difficult for investors to tell what is driving a trend and whether it is fundamentally changing or simply experiencing short-term volatility. For illustration, the price of cryptocurrencies or even stocks might fluctuate dramatically during the day, yet nearly none of this fluctuation reflects a fundamental shift in the security's perceived value. Day traders of cryptocurrencies and stocks trade short-term price movements to enter and exit a position in a matter of minutes or hours. Some noise traders try to profit from market turbulence by entering buy and sell orders without using fundamental data. As a result, this noise may influence the outcome of the study if not dealt with. The aforediscussed points render decomposition an undeniably effective course of action to undertake when dealing with financial time series. We, therefore, decompose the data to illustrate stock market participants' diverse investment time scales which corroborates with the heterogeneous market hypothesis (HMH) as indicated by Müller et al. [38]. Again, the adaptive market hypothesis (AMH) engineered by Lo [40] posits that markets evolve, due to events and structural changes, and adapt, and market efficiency fluctuates in a degree of time. Consequently, decomposition techniques reduce noise (weak signals) to maintain the true signals [10]. This will improve the outcome of the study [33].

This study employs the variational mode decomposition (VMD) method to replace the classic empirical mode decomposition (EMD) method, used for many engineering monitoring and diagnosis. The VMD was developed by Dragomiretskiy and Zosso [41] as a suitable method for sampling and dealing with the noise of signals to surpass EMD and EEMD developed by Huang et al. [42] and $\mathrm{Wu}$ and Huang [43], respectively [44-46]. By shifting from a sifting process to an alternate direction method via multipliers, VMD aids in the solution of the mode mixing problem in decomposition results [41]. Mode mixing, according to $\mathrm{Wu}$ and Huang [47], is defined as any intrinsic mode function (IMF) consisting of oscillations of intensely disparate amplitude, mostly caused by intermittency of the driving mechanism. Thus, the physical meaning of an IMF can cease by itself, indicating that there may be several physical processes in a mode. The IMFs are interpreted as time horizons (amplitude-modulated-frequency-modulated signals). Through the VMD, modes are reasonably extracted nonrecursively, which makes it a fully intrinsic and adaptive and quasi-orthogonal decomposition method [41]. The VMD adaptively determines the suitable band and estimates related modes at the same time, ensuring that the errors between them are suitably balanced [46]. Isham et al. [46] assert that "VMD is based on three principal concepts which are Wiener filtering, Hilbert transform and analytic signal, and frequency mixing and heterodyne demodulation." The VMD meticulously decomposes input signals into their major modes, known as variational mode functions (VMFs), which reproduce the input signal but with varying sparsity qualities. Specifically, in the context of this study, the VMFs represent short-, medium-, and long-term periods. Instead of using a sifting process from the traditional decomposition approach, the VMD approach employs an alternate direction method of multipliers for the reconstruction process [46].

Corollary to the VMD, we present the effective transfer entropy (ETE) that occurs from the formulation of conditional related information [48]. Transfer entropy quantifies the reduction in uncertainty, especially when conditioned on past values in forecasting variables, and thus makes it easier to model statistical causality between variables in a natural phenomenon [49]. Consequently, the amount of information that flows between markets can be quantified using transfer entropy. Therefore, the VMD-based entropy would provide an asymmetric method to measure the flow of information between cryptocurrencies and equities, after accurately decomposing the time series data into their VMFs. This approach is lacking in prior studies on cryptocurrencies 
and stocks $[10,18,50]$. Owing to the complexities in the cryptocurrencies and equities markets coupled with their adaptive behaviours and heterogeneity of their participants, evidence from fractals and multifractality in these markets puts the empirical investigation into perspective $[33,51]$ to reveal investment horizons. As a result, pertinent inferences for portfolio diversification, policy decisions, investing risk, and risk management schemes in cryptocurrencies and global equities could be well accentuated.

The emergence of cryptocurrencies has warranted several studies [10, 18, 50-55]. However, the quantity of information that flows between cryptocurrencies and other financial assets are less captured by prior literature. But, when two random variables are linked in such a way that one can learn about the state of the other by observing the other, they have mutual information [56]. Assessment of this mutual information would provide a clear indication to accurately reflect the benefits of hedges, safe haven, diversification and financial contagion, and the likes. Again, the few empirical studies that focus on the flow of information between cryptocurrencies do not employ decomposition techniques $[7,8,57]$. The current study contributes to prior literature by assessing the flow of information between cryptocurrencies and equities at multiscales, before and during the COVID-19 pandemic.

The novelty of this study relies on the following facets. First, we employ the VMD-based transfer entropy to quantify information flow between global equities and cryptocurrencies by specifying the direction and strength of information transfer at diverse time scales. In this way, we can explore the multiscale information that might be ignored. Also, due to the nonlinearity of most financial time series, we adopt a log-likelihood ratio transfer entropy which quantifies information from a probability density function to fill a gap in the extant literature on cryptocurrencies-equities information flow, which mostly utilises linear models. Despite the popularity of transfer entropy and decomposition techniques in finance and economic literature, there is no study, to the best of our knowledge, which has focused on quantifying the flow of information between global equities and cryptocurrencies while simultaneously dealing with noise through the shift from sifting process via multipliers approach. Furthermore, the major aim of this study is to explore the variation of returns in global equities integration with cryptocurrencies at diverse scales through information flow while incorporating the COVID-19 period. However, analysis of the study would be performed for three sample periods; these are the full sample and pre-COVID-19 and COVID-19 pandemic periods. This would be done to delineate the impact of the COVID-19 pandemic period on financial markets. We utilise six global stock indices with high market capitalization from both NASDAQ and FTSE NAREIT All Equity REITs. This would be done to accurately scrutinize the discussion on cryptocurrencies that have witnessed high market capitalization over the years irrespective of their fall in value in September 2018 [14]. The outcome of the study will bolster confidence in existing investors within these markets to either give up part or all of their investments or ensure their effective management.
We model a mixture of asymmetric and nonlinear bidirectional and unidirectional causality between global equities and cryptocurrencies, for the full sample, as well as before and during the COVID-19 pandemic. However, the COVID-19 period appears to be driving the estimates for the full sample period, which is a negative flow. Thus, the direction and significance of the information flow between the markets for the full sample correspond to the one observed during the COVID-19 pandemic period. We, consequently, establish a significant directional, dynamical, and scale-dependent information flow between global equities and cryptocurrencies. Notwithstanding, throughout the study samples, we mainly find a negative significant flow of information from global equities to cryptocurrencies, in that the history of cryptocurrencies, in this case, indicates considerably more uncertainty than knowing the history of global equities alone. The outcome provides relevant indications to investors with diverse investment horizons who would want to diversify, hedge, or employ cryptocurrencies as a safe haven for global equities during uncertainties [58], specifically the COVID-19 pandemic.

The remaining sections are systematized as follows. Section 2 deals with literature review issues, Section 3 with research methodology, and Section 4 with the study's findings and discussion. Section 5 contains the study's conclusion, which includes the findings' implications and suggestions.

\section{Literature Review}

2.1. Theoretical Review. We present three theories to expand the discussion on the flow of information between global equities and cryptocurrencies. These theories are explained concerning portfolio selection by investors as advised by Markowitz's [37] modern portfolio theory. The theories that guide the study variables include equity parity theory, theory on interdependence, and information flow theory.

2.1.1. Modern Portfolio Theory. The modern portfolio theory of Markowitz [37] asserts that investors tend to either maximise their expected return or minimise the variance on their portfolio. The input of a security to the variance of an investor's entire portfolio, according to Markowitz [37], is more relevant than the security's own risk. This is mainly a question of its covariance with other securities in the portfolio [59]. In the context of this study, an increase in trading volumes of cryptocurrencies attracts investors to the cryptocurrencies markets [60]. As a result of the accumulation of cryptocurrencies in major market players' investment portfolios, prices will stabilize and uncertainty will decrease [61]. Also, this relationship may not be far from stock markets [62]. The study, therefore, seeks to analyse the extent to which investments in both cryptocurrencies and stocks markets could earn investors with diversification, safe haven, and hedge benefits, before and during the COVID-19 pandemic [58]. 
2.1.2. Equity Parity Theory. This emerging theory in international finance suggests the connection between equities and currency movements. According to this theory, when investors' foreign or international holdings outperform domestic holdings, domestic investments are exposed to higher exchange rates which devalue domestic currencies contributing to inflation [63-65]. This is facilitated by the fact that an increase in trading volumes attracts investors as posited by Ji et al. [60]. The inflationary pressures are mitigated by higher interest rates which plummet domestic assets values in the long-run [66]. Furthermore, to decrease exchange rate risks, domestic investors may repatriate part of their foreign holdings as a reaction to adverse movements in domestic currencies [67]. By doing so, foreign currency is sold contributing to its depreciation. In the context of this study, the equity parity theory addresses the extent to which fluctuations in cryptocurrencies correspond to fluctuations in stocks. That is, as individuals invest in cryptocurrencies as an international holding, domestic equity holdings are exposed to adverse exchange rates shocks. The global equities used in this study are therefore reflective of domestic equity holdings across countries.

2.1.3. Theory on Interdependency. Interdependence is defined as the situation whereby there is a direct link between assets (markets), for instance, cryptocurrencies and stock markets, such that when there is a change in the direction of one state, it influences the direction of the others in the same way. The study follows horizontal interdependence which considers the flow of money or capital from one market to another, in the context of this study. There are several concepts on economic interdependence. For instance, Doyle [68] postulated that when there is interdependence, it results in increased economic relations among countries which promotes the forming of unions among those countries. Polachek [69] argues that countries gain from interdependence, for example, the diverse advantages obtained from trading with other nations which most governments try to sustain. Furthermore, interdependence may suggest common values in trading or socio-cultural ethos which involve the maintenance of international order. The study extends these arguments to illustrate the interdependence between markets, specifically nontraditional markets (cryptocurrencies) and global equities markets. It further indicates the extent to which investors of union countries' stocks may simultaneously benefit from adding cryptocurrencies.

2.1.4. Information Flow Theory. Benthall [56] advocates that information flows are causal flows located in the context of other causal linkages. Information flow theory builds on the philosophy of Dretske [70] and the statistics of Pearl [71]. The mathematics of probability and statistics has made it possible to quantify the information flow between variables. Intuitively, reciprocal information exists when two random variables are linked in such a way that one variable can learn about the state of the other from observation of the other
[56]. As indicated by Ji et al. [60], an increase in trading volumes of cryptocurrencies attracts investors to the cryptocurrencies market due to higher expectations of price stability and minimal uncertainty.

Similarly, Umar et al. [72] posited that cryptocurrencies gained worldwide attention in 2017 after a significant increase in their value, resulting in investors shifting a large portion of their assets from traditional financial assets to cryptocurrencies. The price increase, however, was not sustainable, and the cryptocurrency bubble burst at the end of 2018 [72]. Investors of cryptocurrencies who are riskaverse may consider cryptocurrencies as very risky and may channel their investments to less risky traditional financial assets such as stocks [73]. It goes to suggest that the creation of new assets (markets) contributes to competitive alternative markets through which investors can channel part or all of their investments based on their level of risk aversion. This establishes the flow of information between the markets.

2.2. Empirical Review. The emergence of cryptocurrencies has warranted several studies. Most studies either concentrate on cryptocurrencies in isolation [52-54] or combined with other assets $[10,18,50,51]$. Prior studies that combine cryptocurrencies with other assets mostly look out for hedge, safe haven, and diversification opportunities $[10,15,18,51]$; spillover sentiments which are greatly influenced by major market events [50]; and financial contagion of cryptocurrencies [55]. These studies assess either relationships or causality between and among financial assets without employing the transfer entropy.

This is to say, the quantity of information that flows between cryptocurrencies and other financial assets are less captured by prior literature. But, when two random variables are linked in such a way that one can learn about the state of the other by observing the other, they have mutual information [56]. Assessment of this mutual information would provide a clear indication to accurately reflect the benefits of hedges, safe haven, diversification, spillover sentiments and financial contagion, and the likes.

Again, the few studies that assess the flow of information between cryptocurrencies and other financial assets do not consider decomposition techniques [7, 8, 57]. However, market participants react to information at different times, resulting in very noisy market data. To correctly delineate this issue, the presence of decomposition techniques in this study would proffer stock market participants' diverse investment time scales. This is in line with the heterogeneous market hypothesis (HMH) as indicated by Müller et al. [38]. Also, the adaptive market hypothesis (AMH) indicated by Lo [40] suggests that markets evolve, due to events and structural changes, and adapt, and market efficiency fluctuates in the degree of time. Consequently, decomposition techniques would minimise weak signals [10]. Accordingly, quantifying information flow between cryptocurrencies and global equities at multiscales, before and during the COVID19 pandemic, would be of principal interest to market agents. 


\section{Methodology}

We initially present the VMD technique, followed by the transfer entropy. Thus, the outcome generated from the VMD is used as input data for the ETE estimations.

3.1. VMD. An amplitude-modulated-frequency-modulated signal is defined as the intrinsic mode functions (IMF) of the VMD [41]. The $k_{\text {th }}$ mode $u_{k}(t)$ is presented as

$$
u_{k}(t)=A_{k}(t) \cos \left(\phi_{k}(t)\right)
$$

where $A_{k}(t)$ is the immediate amplitude; $\phi_{k}(t)$ is the immediate phase; its derivative $\omega_{k}(t)=\phi_{k}(t)$ is the immediate scale.

For each mode $u_{k}(t)$, VMD uses the Hilbert transform to produce the analytic signal and estimates the autonomous frequency spectrum. The spectrum of the mode is then moved to baseband using the Fourier transform's displacement property. Subsequently, the bandwidth is projected via the $H^{1}$ Gaussian smoothness. The purpose of optimization is to reduce the sum of all mode functions' spectral widths to the smallest possible value as

$$
\begin{aligned}
& \min _{\left\{u_{k}\right\},\left\{\omega_{k}\right\}}\left\{\sum_{k=1}^{k}\left\|\partial_{t}\left[\left(\delta(t)+\frac{j}{\pi t}\right) * u_{k}(t)\right] e^{-j \omega_{k} t}\right\|_{2}^{2}\right\}, \\
& \text { s.t. } \sum_{k=1}^{k} u_{k}=f,
\end{aligned}
$$

where $\left\{u_{k}\right\}$ is the mode ensemble; $\left\{\omega_{k}\right\}$ is the analogous centre frequency ensemble; $K$ is the mode observation. The original signal is equal to the total of the modes, which is the constraint.

A quadratic penalty term and a Lagrangian multiplier are introduced to change the preceding constrained optimization problem into an unrestrained issue as follows:

$$
L\left(\left\{u_{k}\right\},\left\{\omega_{k}\right\}, \lambda\right)=\alpha \sum_{k=1}^{k}\left\|\partial_{t}\left[\left(\delta(t)+\frac{j}{\pi t}\right) * u_{k}(t)\right] e^{-j \omega_{k} t}\right\|_{2}^{2}+\left\|f(t)-\sum_{k=1}^{k} u_{k}(t)\right\|_{2}^{2}+\lambda(t), f(t)-\sum_{k=1}^{k} u_{k}(t)
$$

where $\quad \alpha$ is the penalty parameter; $\lambda$ is the Lagrangian multiplier.

The alternating direction method of multipliers (ADMM) is used by VMD to solve the above equation iteratively. Finally, the original signal is decomposed into $K$ IMF constituents. The code of VMD is available in Hamilton and Ferry's [74] package "VMD."

3.2. Renyi Transfer Entropy. Before we discuss the Rényi transfer entropy, we present the idea of Shannon entropy as a quantification of uncertainty upon which transfer entropy is embedded in information theory $[49,75]$. We consider a probability distribution with diverse results of a given experiment $p_{j}$. The average of each symbol's information is specified as

$$
H=\sum_{j=1}^{n} P_{j} \log _{2}\left(\frac{1}{P_{j}}\right) \text { bits, }
$$

where $n$ signifies number of distinct symbols concerning the probabilities $P_{j}$.

The concept of entropy was introduced in 1948 by Shannon [76]. It proffers that, for a discrete random variable $(J)$ with probability distribution $(P(j))$, the average number of bits needed to maximally encode independent draws [75] can be presented as

$$
H_{J}=-\sum_{j=1}^{n} P(j) \log _{2} P(j) .
$$

Shannon entropy is connected with the concept of Kullback-Leibler distance [77] to assess the information flow between two-time series with the concept of Markov processes. We present $I$ and $J$ as two discrete random variables with corresponding marginal probabilities of $P(i)$ and $P(j)$, joint probability $P(i, j)$, with dynamic structures in line with a stationary Markov process of order $k$ (Process $I$ ) and $I$ (process $J$ ). The Markov property implies that the probability to observe $I$ at time $t+1$ in state $i$ conditional on the $k$ prior observations is $p\left(i_{t+1} \mid i_{t}, \ldots\right.$, $\left.i_{t-k+1}\right)=p\left(i_{t+1} \mid i_{t}, \ldots, i_{t-k}\right)$. To encode the observation in $t+1$, the average bits number required once the ex-ante $k$ values are known can be illustrated as

$$
h_{j}(k)=-\sum_{i} P\left(i_{t+1}, i_{t}^{(k)}\right) \log P\left(i_{t+1} \mid i_{t}^{(k)}\right),
$$

where $i_{t}^{(k)}=\left(i_{t}, \ldots, i_{t-k+1}\right)$ (correspondingly for process $\left.J\right)$. In a bivariate perspective as well as relying on the Kullback-Leibler distance, information transmission from process $J$ to process $I$ is measured by quantifying the deviation from the generalized Markov property $P\left(i_{t+1} \mid i_{t}^{(k)}\right)=P\left(i_{t+1} \mid i_{t}^{(k)}, j_{t}^{(I)}\right)$. The Shannon transfer entropy can thus be presented as

$$
T_{J \longrightarrow I}(k, l)=\sum P\left(i_{t+1}, i_{t}^{(k)}, j_{t}^{(I)}\right) \log \frac{P\left(i_{t+1} \mid i_{t}^{(k)}, j_{t}^{(I)}\right)}{P\left(i_{t+1} \mid i_{t}^{(k)}\right)}
$$

where $T_{J \longrightarrow I}$ calculates the information flow from $J$ to $I$. Analogously, $T_{I \longrightarrow J}$, as a measure for the information flow from $I$ to $J$, can be derived. Calculating the differential can reveal the prevalent direction of information flow between $T_{J \longrightarrow I}$ and $T_{I \longrightarrow I}$.

Based on the Shannon entropy so far discussed, we present the Rényi Transfer Entropy [78] which is contingent on a weighting factor $q$ and can be calculated as 


$$
H_{J}^{q}=\frac{1}{1-q} \log \sum_{j} P^{q}(j)
$$

with $q>0$. For $q \longrightarrow 1$, Rényi entropy converges to Shannon entropy. For $0<q<1$, thus, low probability events receive more weight, while for $q>1$, the weights benefit outcomes $j$ with a higher original probability. As a result, Rényi entropy permits to accentuate diverse distribution areas, depending on factor $q[49,75]$.

Applying the escort distribution [79] $\varnothing_{q}(j)=\left(p^{q}(j) /\right.$ $\left.\sum_{j} p^{q}(j)\right)$ with $q>0$ to normalize the weighted distributions, Rényi transfer entropy [78] is derived as

$$
R T_{J \longrightarrow I}(k, l)=\frac{1}{1-q} P\left(i_{t+1}, i_{t}^{(k)}, j_{t}^{(I)}\right) \log \frac{\sum_{i} \varnothing_{q}\left(i_{t}^{(k)}\right) P^{q}\left(i_{t+1} \mid i_{t}^{(k)}\right)}{\sum_{i, j} \varnothing_{q}\left(i_{t}^{(k)}, j_{t}^{(I)}\right) P^{q}\left(i_{t+1} \mid i_{t}^{(k)}, j_{t}^{(I)}\right)} .
$$

It is worth noting that the Rényi transfer entropy calculation can have negative results. Knowing the history of $J$, in this case, indicates considerably more uncertainty than knowing the history of $I$ alone would indicate.

The ETE estimates are biased in small samples [80]. The correction of the bias is promising and can be applied to calculate the ETE as

$$
\operatorname{ETE}_{J \longrightarrow I}(k, l)=T_{J \longrightarrow I}(k, l)-T_{\text {Jshuffled } \longrightarrow I}(k, l),
$$

where $T_{\text {Jshuf fled } \longrightarrow I}(k, l)$ depicts the transfer entropy using a shuffled version of the time series $J$ that is randomly drawing values from the observed time series $J$ and readjusting them to generate a new time series. This, therefore, destroys the time series dependencies of $J$, not forgetting the statistical dependencies between $J$ and $I$. This enjoins $T_{\text {Jshuffled } \longrightarrow I}(k, l)$ to come together to zero with increasing sample size, and any nonzero value of $T_{\text {Jshuffled } \rightarrow I}(k, l)$ is due to small sample effects. To produce a bias-corrected ETE estimate, repeated shuffling and the average of the shuffled transfer entropy estimates overall replications indicates a predictor for the small sample bias. This is, thus, removed from the Shannon or Rényi ETE estimate.

Relying on a Markov block, the significance of the transfer entropy measures from equation (10) can be inspected, as provided by [81]. This preserves the interconnectedness within the variables $J$ and $I$, but ignores the statistical dependencies between $J$ and $I$ opposing to shuffling. The distribution of the estimates under the null hypothesis of no significant information transmission is then determined. This can be done by repeated estimation of the transfer entropy. The associated $p-$ value is given by $1-\hat{q} T$, where $\hat{q} T$ signifies the simulated distribution's quantile, which is defined by the transfer entropy estimate [75].

3.3. Empirical Data. The study employed daily prices which are made up of NASDAQ Global Market Composite Index (GMI), Eurozone Index (EUROZI), Europe Index (EUROPI), Emerging Markets Index (EMI), and Developed Markets Index (DMI); Global Real Estate Investment Trust Equities (GREITs) measured as FTSE NAREIT All Equity REITs; and cryptocurrencies Bitcoin (BTC), Dash, Litecoin (LTC), and Ripple (XRP). The daily data span from 2014/09/18 to 2021/ 05/07 yielding a total of 1,589 observations after merging the data to have a common date. The suggested time frame was chosen to encompass the US-China trade war, the birth of COVID-19 as a global pandemic, and the aftermath of the 2007-2009 global financial crisis. The global equity indices are carefully selected to ascertain the aggregated effect of equities to illustrate their information flow with cryptocurrencies as they compete to reveal the competitive market hypothesis [33]. However, the cryptocurrencies were chosen for the given time frame based on continuous data availability with large market capitalization. In addition, the cryptocurrencies selected are mostly employed by extant literature to examine the extent of diversification, safe haven, and hedge benefits with other assets. This is so because, the largest cryptocurrency, Bitcoin, has experienced growing competition from other cryptocurrencies which has heightened the heterogeneity in these markets [51]. Because financial data fluctuates rapidly, daily data was chosen over monthly series [82]. The data on NASDAQ equities were obtained from EquityRT whilst those of GREITs and cryptocurrencies were obtained from Yahoo Finance, with the US dollars as the currency value. As shown below, the study was based on daily returns of $r_{t}=\ln P_{t}-\ln P_{t-1}$, where $r_{t}$ is the continuously compounded return and $P_{t}$ and $P_{t-1}$ are current and preceding index, respectively.

\section{Results and Discussion}

4.1. Descriptive Analysis. Figure 1 provides the time-varying prices and returns of both global equities and cryptocurrencies. It can be observed from the plots that in the early part of 2020, the price series for all markets trend upwards after a downward spike. That is, the prices of both cryptocurrencies and global equities are experiencing a rapid increase which concurs with the assertion made by Zhang et al. [83] of markets rebound later in the COVID-19 periods since most businesses and economies have learnt how to survive. Generally, it can be observed from the plots that fluctuations in cryptocurrencies supersede those of global equities. This supports the assertion made by extant literature on the riskiness of cryptocurrencies as against equities $[15,72,73]$.

Table 1 exhibits the summary of statistics for the markets during the period under study. The skewness values observed show nonnormality across board with Dash exhibiting a skewness close to normality of 0.4037 . On the other hand, 


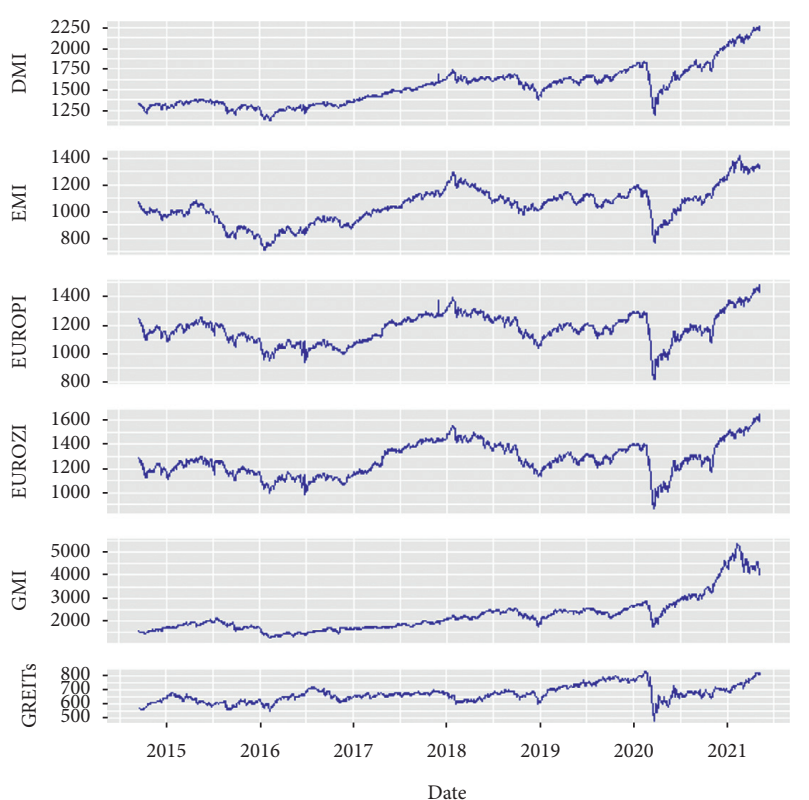

(a)
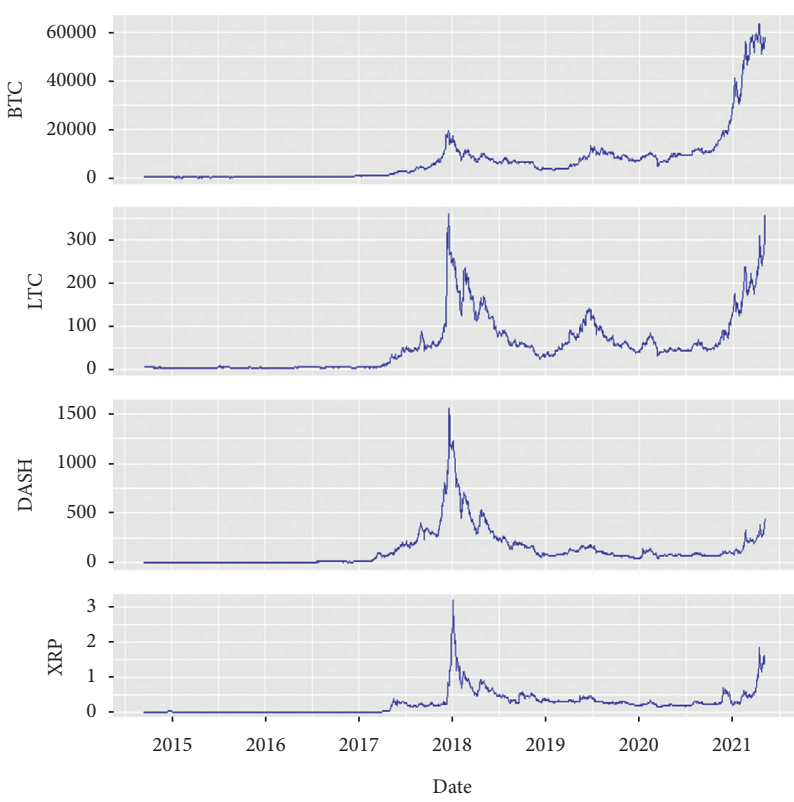

(c)

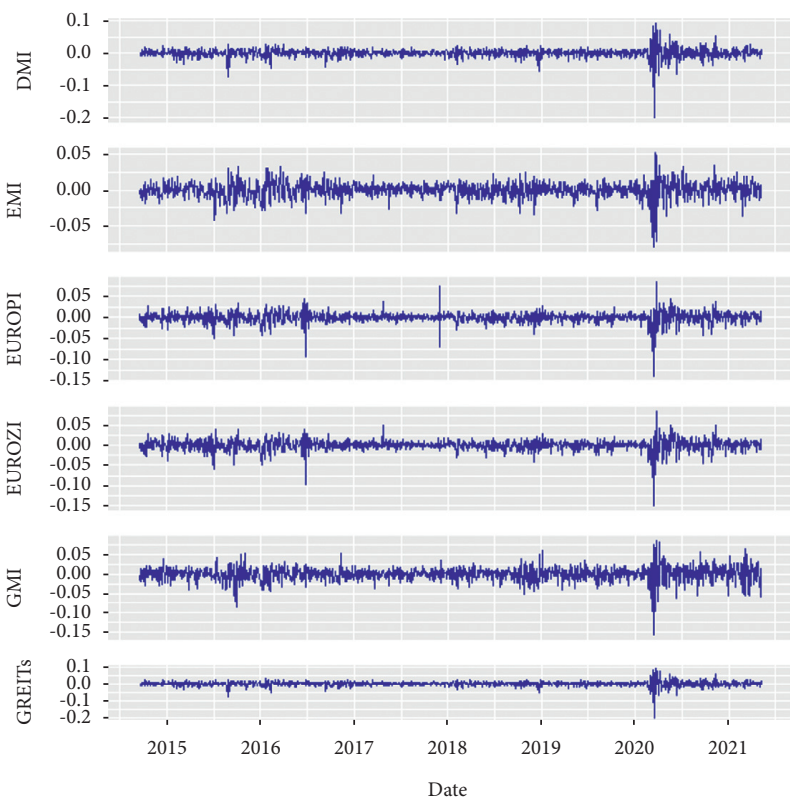

(b)
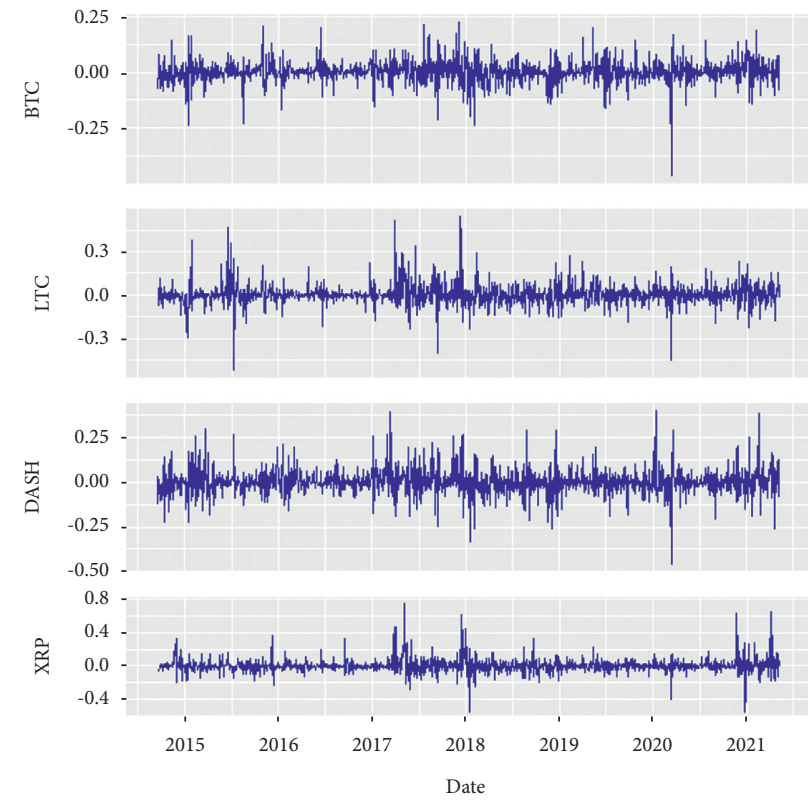

(d)

FIgURE 1: Plots of price and returns series for both global equities and cryptocurrencies.

kurtosis values further show leptokurtic behaviour in the values across variables, especially gross domestic product. In terms of the stationarity test, the augmented Dicky-Fuller (ADF) and the Kwiatkowski-Phillips-Schmidt-Shin (KPSS) tests are used. The observations from both the ADF and the KPSS reveal that all the data series explicitly fulfil the stationarity requirements. This is in line with assumptions of various autoregressive studies such as $[84,85]$ which assumes global stationarity. Despite the stationarity of the time series returns, we respond further to dormant nonstationarity, asymmetry, and nonlinearity by employing the VMD-based ETE in the context of the study.
4.2. Empirical Results. The empirical results are presented for three sample periods. The first section deals with the full sample. This is followed by period-specific analyses indicating both before and during COVID-19 pandemic period dimensions. To fully reflect the power of the VMD-based entropy, we present the multiscales obtained from decomposing the time series for each scenario to get more insights into the time variability of the flows and further deal with issues of nonlinearity, nonstationarity, and asymmetry which may occasion a deterministic system to chaos [86]. In this study, the signal depicts the original time series from which the decompositions are calculated. The M1, M2, M3, 
TABLE 1: Descriptive summary.

\begin{tabular}{|c|c|c|c|c|c|c|c|}
\hline & Mean & Std. Dev & Skewness & Kurtosis & Normtest.W & $\mathrm{ADF}$ & KPSS \\
\hline \multicolumn{8}{|c|}{ Global equities } \\
\hline DMI & 0.0003 & 0.010 & -1.444 & 20.536 & 0.824 & $-11.087^{* * *}$ & 0.128 \\
\hline EMI & 0.0001 & 0.010 & -0.937 & 7.623 & 0.928 & $-10.606^{* * *}$ & 0.136 \\
\hline EUROPI & 0.0001 & 0.012 & -1.524 & 18.755 & 0.860 & $-11.802^{* * *}$ & 0.116 \\
\hline EUROZI & 0.0002 & 0.013 & -1.626 & 18.157 & 0.876 & $-11.578^{* * *}$ & 0.100 \\
\hline GMI & 0.0006 & 0.017 & -0.966 & 8.726 & 0.920 & $-11.193^{* * *}$ & 0.097 \\
\hline GREITs & 0.0002 & 0.014 & -2.339 & 36.378 & 0.786 & $-11.362^{* * *}$ & 0.026 \\
\hline \multicolumn{8}{|c|}{ Cryptocurrencies } \\
\hline BTC & 0.0030 & 0.048 & -0.634 & 9.292 & 0.899 & $-10.535^{* * *}$ & 0.183 \\
\hline Dash & 0.0029 & 0.070 & 0.404 & 5.375 & 0.916 & $-10.493^{* * *}$ & 0.172 \\
\hline LTC & 0.0027 & 0.070 & 0.789 & 11.918 & 0.843 & $-9.8306^{* * *}$ & 0.135 \\
\hline XRP & 0.0036 & 0.0813 & 1.6913 & 17.5021 & 0.784 & $-10.149^{* * *}$ & 0.085 \\
\hline
\end{tabular}

Note: Normtest.W indicates a significant difference from a normal distribution at all conventional levels of significance. ${ }^{*},{ }^{* *}$, and ${ }^{* * *}$ indicate significance at $10 \%, 5 \%$, and $1 \%$ levels, respectively.

and MAgg are the decomposed time series. The first three VMFs (M1, M2, and M3) are the simulated signals whilst the fourth VMF (MAgg) is the Gaussian white noise added to the signal. We represent M1 to be the short-term, M2 and M3 signify medium-terms, and MAgg shows the long-term trend (fundamental feature). For simplicity, we present a brief discussion of pertinent scales and developed relationships that may be of importance to market participants.

4.2.1. Full Sample Analysis. From Figure 2 (the transfer entropies for Figures 2-4 have been provided in a table form as Tables 2-4), most of the information flows between global equities and Bitcoin are insignificant at diverse time scales, except for the short-term (flow towards Bitcoin). From the original series (signal), Bitcoin transmits significant information to the Emerging Market Index (EMI). Generally, flows towards Bitcoin are more negative than flows towards equity. This signifies that information flow from global equities transmits negative shocks to the Bitcoin market at diverse investment horizons. In addition, the original series depicts a positive flow between global equities and Bitcoin, but a more negative flow was found at diverse investment horizons. This shows that the original return series of the markets of Bitcoin and global equities try to observe the behaviour of each other directly with minimal uncertainty. However, since market participants react to information at different times, the multiscales reveal negative information flow, especially in the short- and medium-terms. This is in line with the findings of Jang et al. [87] who found an asymmetric flow of information from other investment assets to Bitcoin. For the sake of asset allocation and risk management, the negative information flow between Bitcoin and global equities at various investment horizons brings the findings of Zhang et al. [88] to light.

The outcome of Bitcoin is not far from Dash, which shows the information flow between global equities and Dash returns. However, there are traces of significant information flow at all scales as compared to Bitcoin. Since the market capitalization of Dash is currently low, investors who seek to maximise their returns would rather divest from Dash and invest in equities. That is a significant negative flow to Dash market may lead to its downturn. This can be examined from the positive flow from Dash to most global equities. However, the significant negative flow (M2, M3, and MAgg) from Dash to the global market index shows that investors with medium-to-long-term holdings of Dash may scale up their investments with the hope of earning higher future returns.

The findings from Figure 2 show that in the short- and medium-terms, there are negative flows of information from global equities to Litecoin. Thus, investors who hold their investments in Litecoin for a long-term receive positive information from global equities. Contrarily, Litecoin transmits negative information to global equities, especially in the short-term with a significant flow to the Emerging Market Index, Eurozone Index, and Europe Index.

Moreover, Figure 2 illustrates the dynamics of both positive and negative significant flow of information from global equities to Ripple. That is, in addition to the potential for tail events dynamics of the Rényi transfer entropy as shown by the original series (signal), multiscales perspectives also provide adequate information to delineate the information theory. This corresponds to the heterogeneous market hypothesis [38] where market participants respond differently to information in the markets at diverse investment horizons.

4.2.2. Pre-COVID-19 Pandemic Period. We further present a confirmatory analysis of the original return series to establish the flow of information between global equities and cryptocurrencies before and during the COVID-19 pandemic. We take cognisance of the World Health Organization's (WHO) communiqué, which designated the COVID-19 outbreak a Public Health Emergency of International Concern (PHEIC) on January 30, 2020, and a pandemic on March 11, 2020. We, therefore, segregate the return series based on the declaration by the WHO on $30^{\text {th }}$ January 2020 as a PHEIC to inculcate early investors' expectations and fear during the pandemic.

Generally, from the pre-COVID-19 pandemic period, there seem to be some positive flows from global equities in addition to the usual negative flows established from the full 


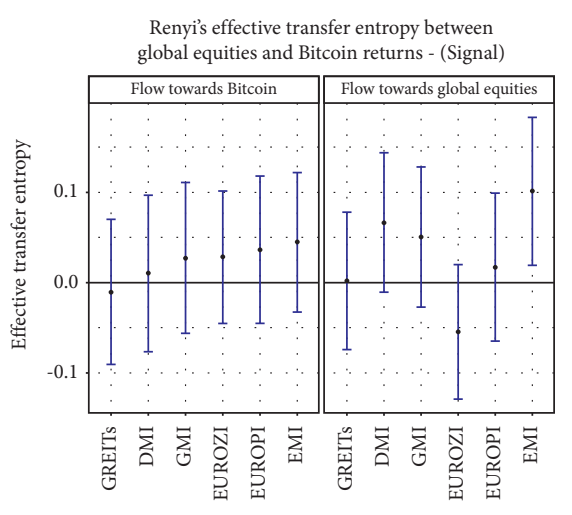

(a)

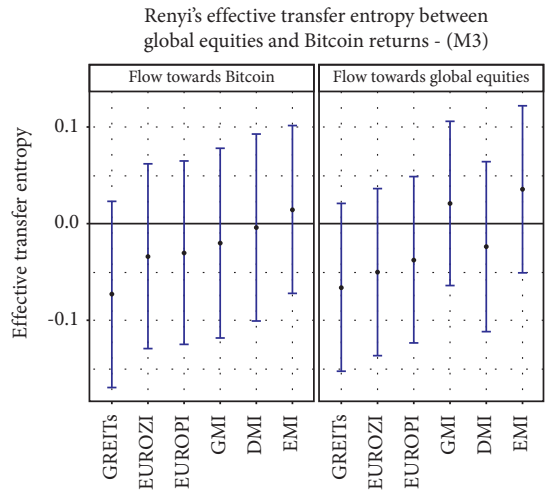

(d)

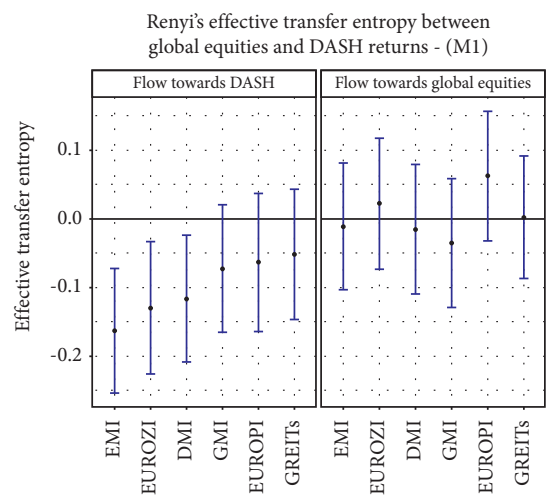

(g)

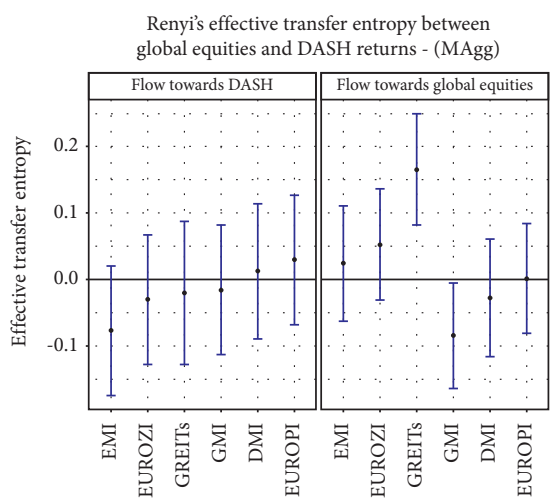

(j)

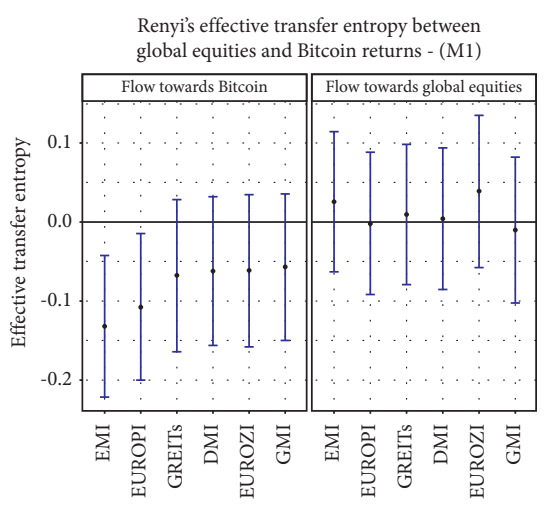

(b)

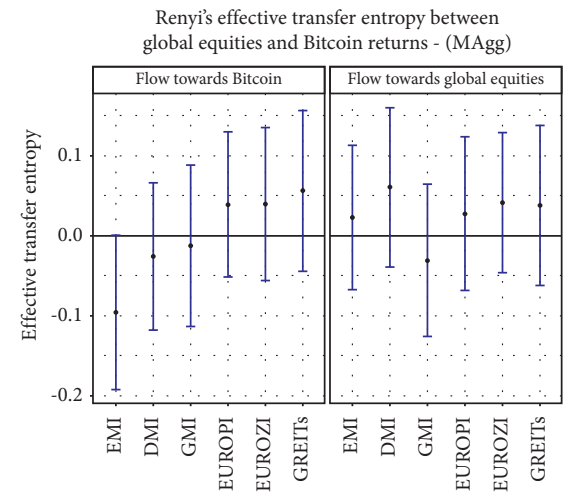

(e)

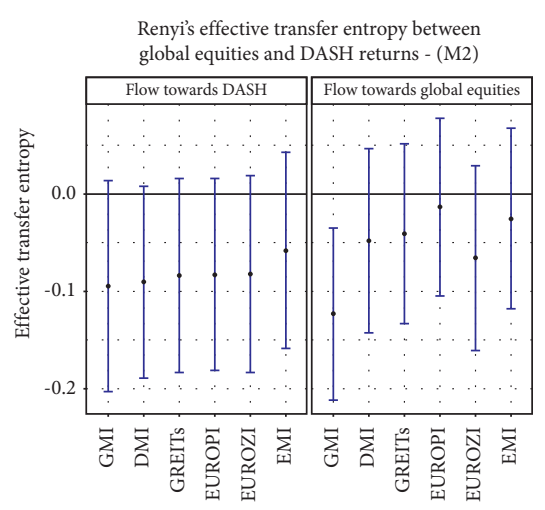

(h)

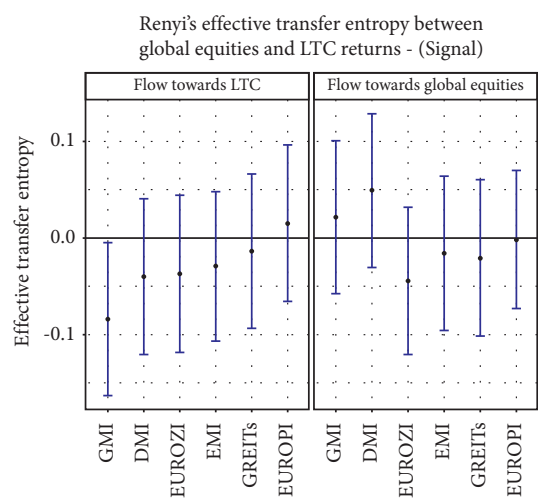

(k)

Figure 2: Continued.

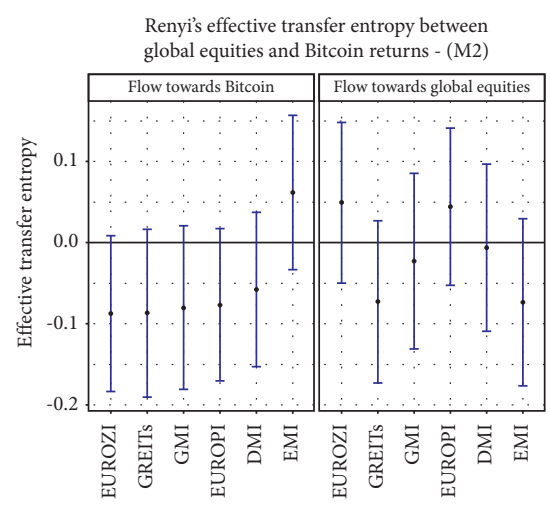

(c)

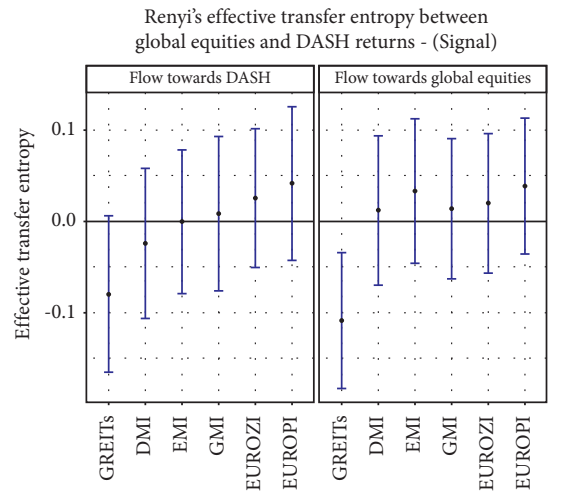

(f)

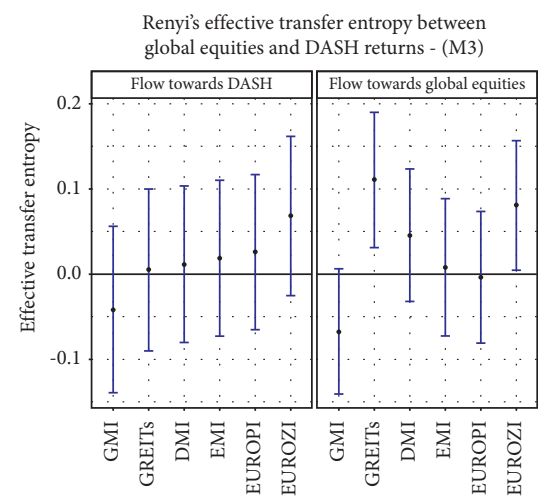

(i)

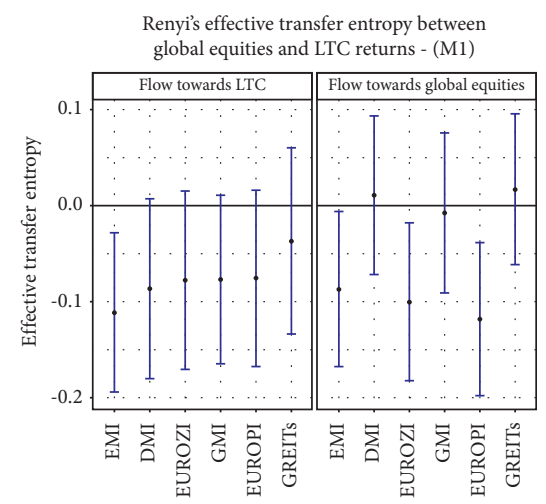

(1) 


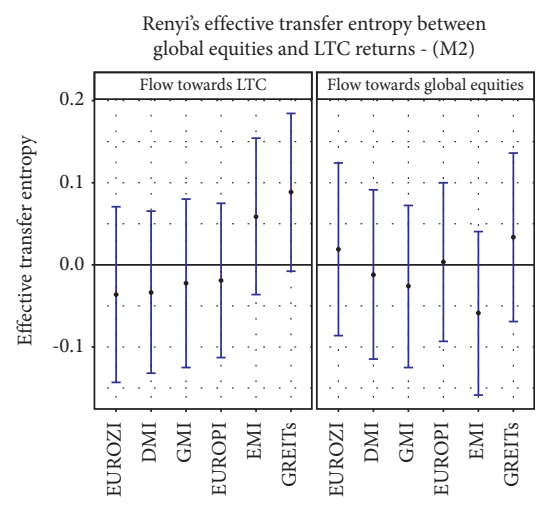

$(\mathrm{m})$

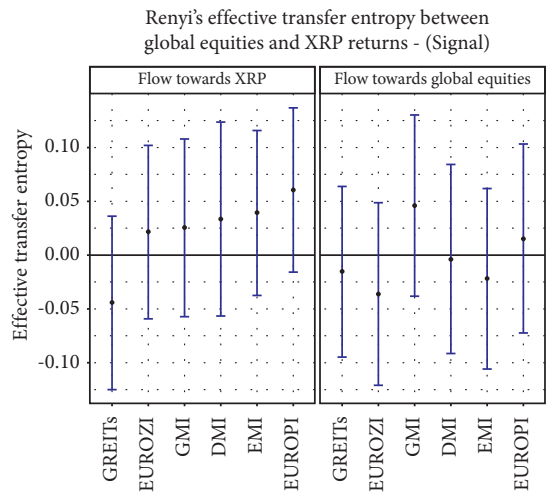

(p)

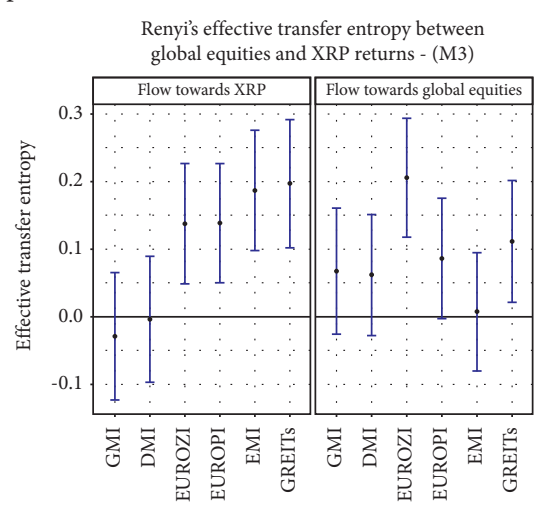

(s)

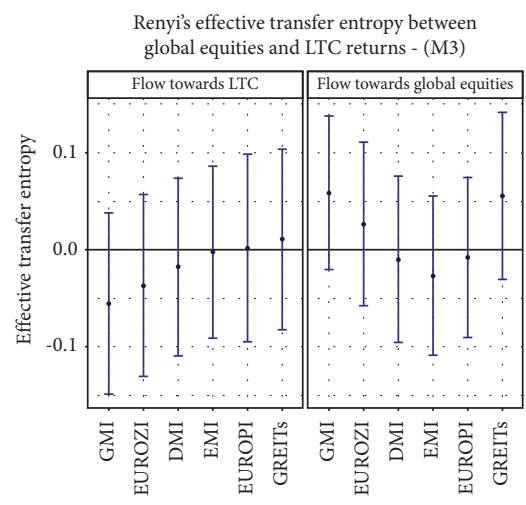

(n)

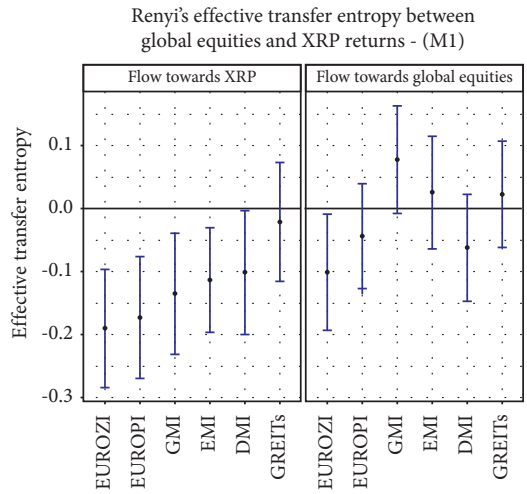

(q)

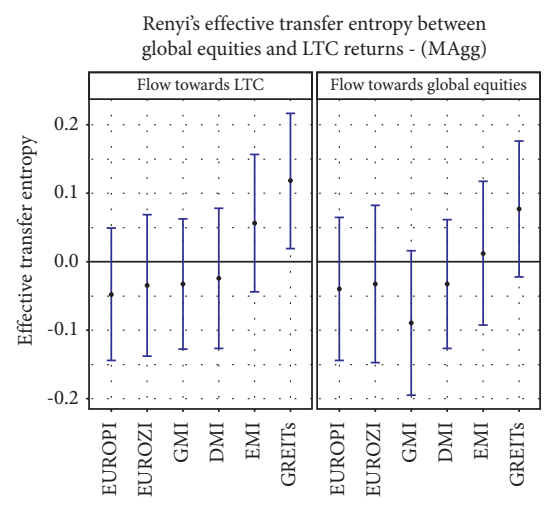

(o)

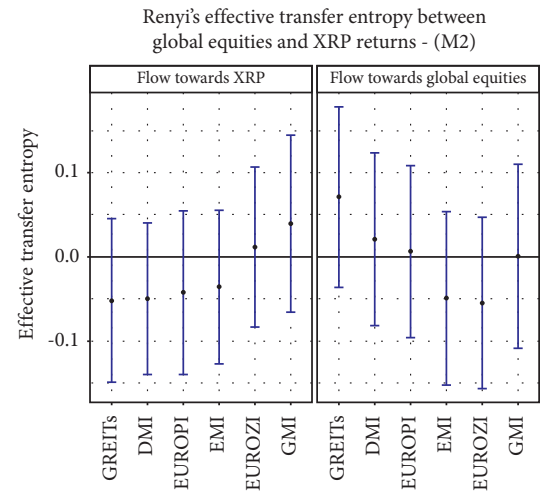

(r)

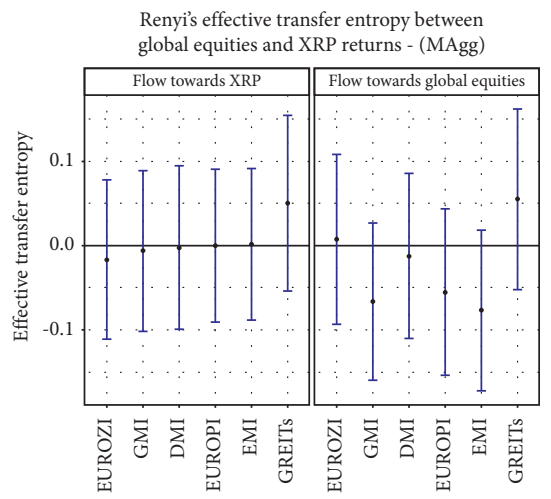

$(\mathrm{t})$

Figure 2: Information flow between global equities and cryptocurrencies (full sample period).

sample case. That is, the patterns of information flow have altered when the COVID-19 pandemic period was screened out from the full sample. Specifically, from Figure 3, there are both positive and negative flows from global equities to Bitcoin, and the reverse is also true. Notwithstanding, there are more negative flows than there are positive flows which establish the asymmetric information flow by Jang et al. [87]. Also, the patterns of information flow within each scale do not seem to deviate significantly from each other, which establishes the long-run memory in Bitcoin [89].

From Figure 3, the patterns of information flow from global equities to Dash and from Dash to global equities do not change substantially from one scale to the other. Generally, Dash receives more negative flow from global equities, but transmits a mixture of both positive and negative flow. This is similar to the Bitcoin from the preCOVID-19 period.

Also, negative information flows from global equities to Litecoin except for flows from the Global market index and global real estate index in the medium and long-term perspectives. That is, there are higher likelihoods of Litecoin serving as a hedge for global equities, except for the Global Market Index and Global Real Estate Index at certain time horizons (M2 and MAgg).

From Figure 3, the scales (M1-MAgg) significantly differ from the original series concerning flow from global equities to Ripple. However, among the scales, the signs of future orders are predicted from the signs of historical orders which 


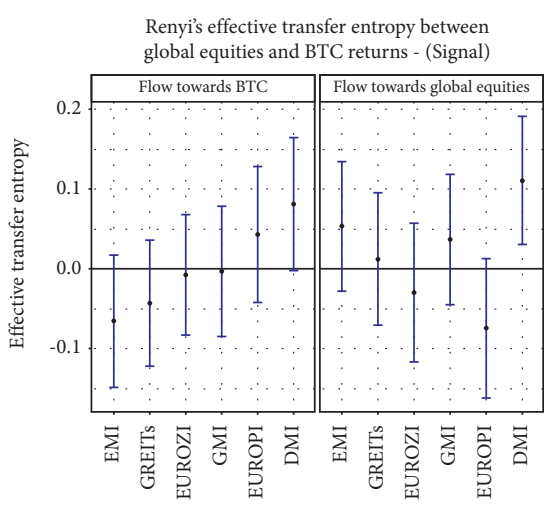

(a)

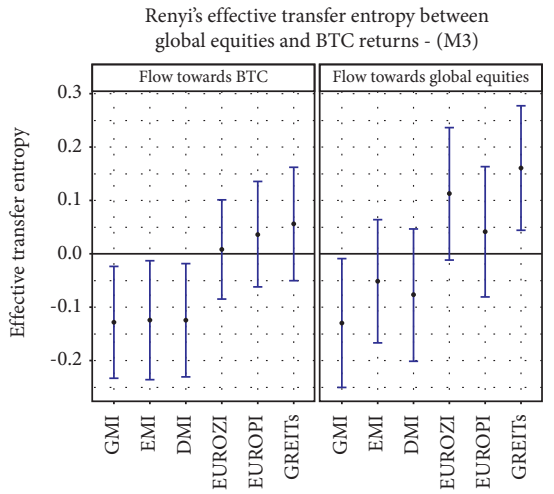

(d)

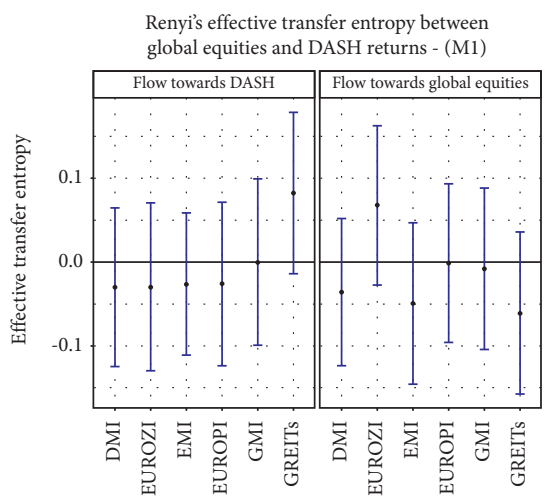

(g)

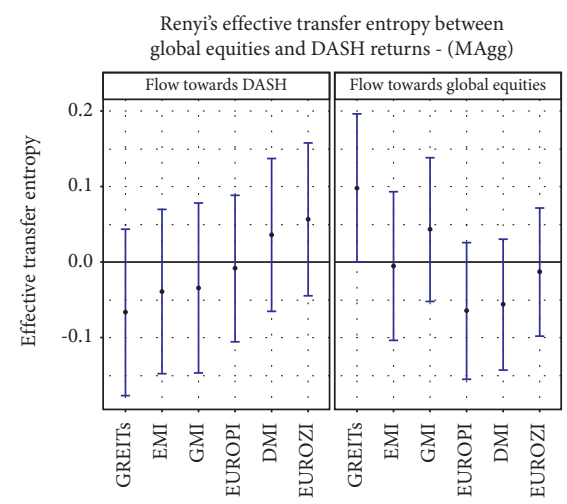

(j)

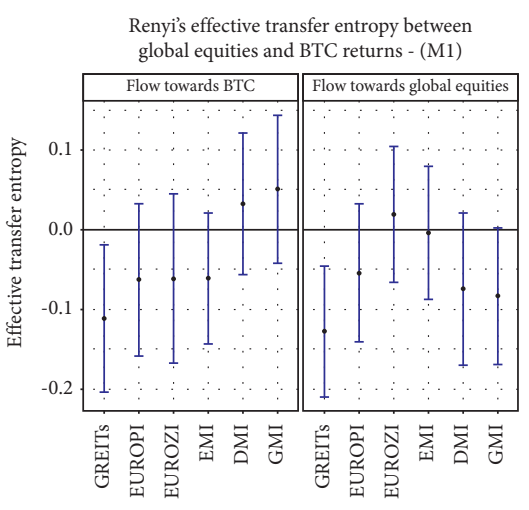

(b)

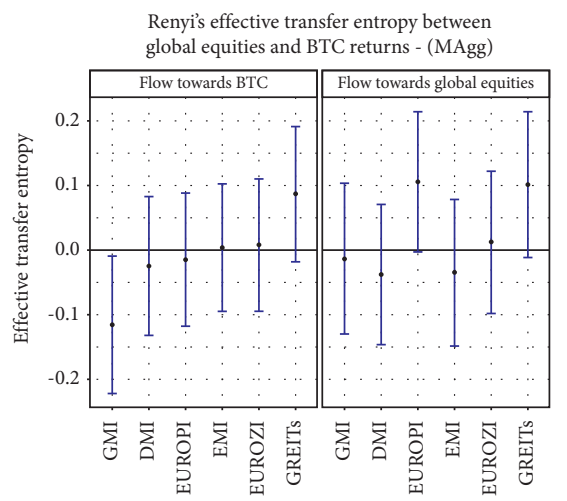

(e)

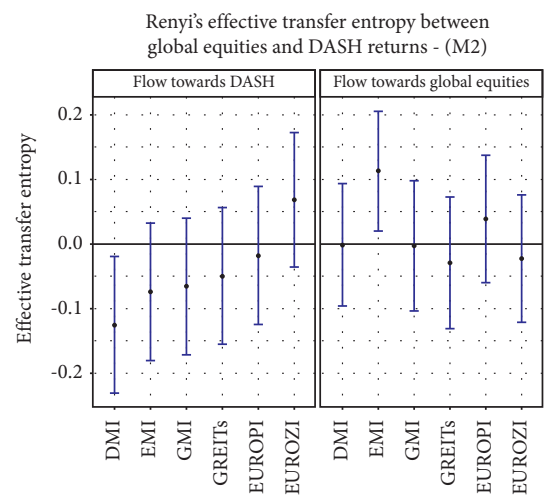

(h)

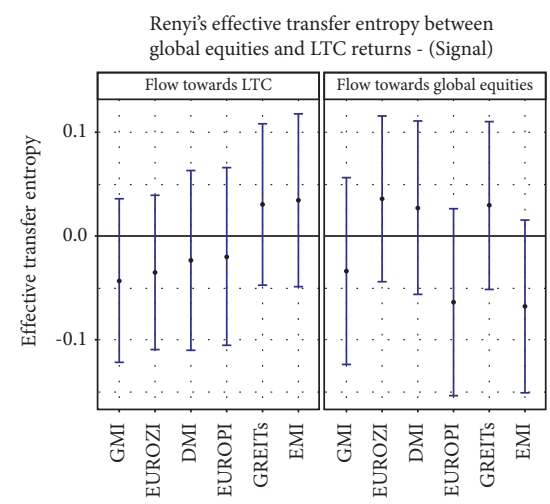

(k)

Figure 3: Continued.

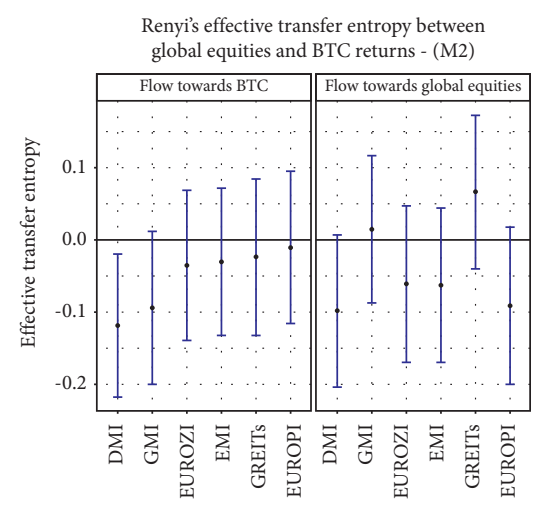

(c)

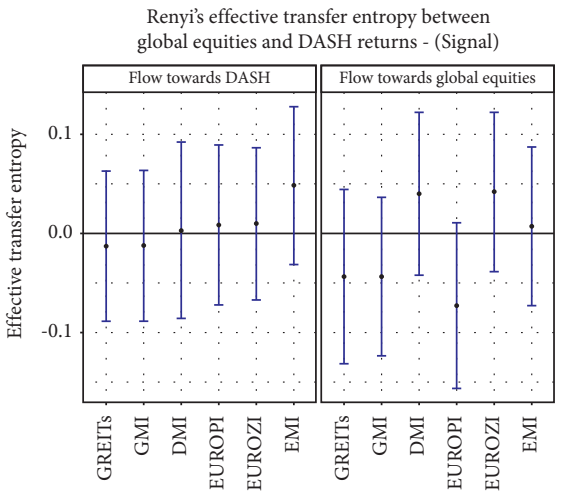

(f)

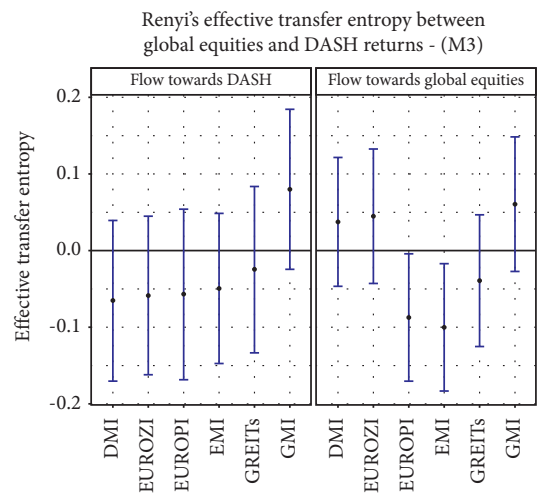

(i)

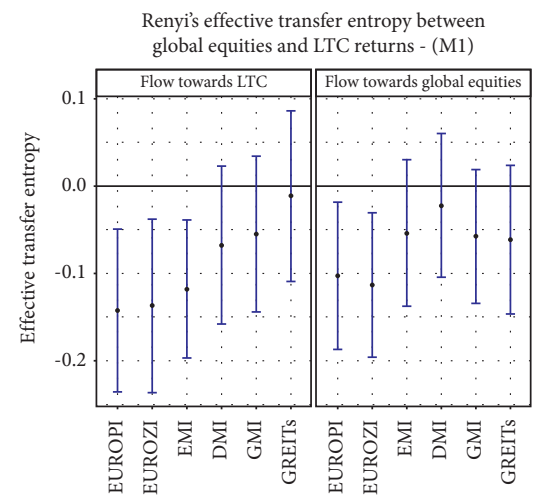

(1) 


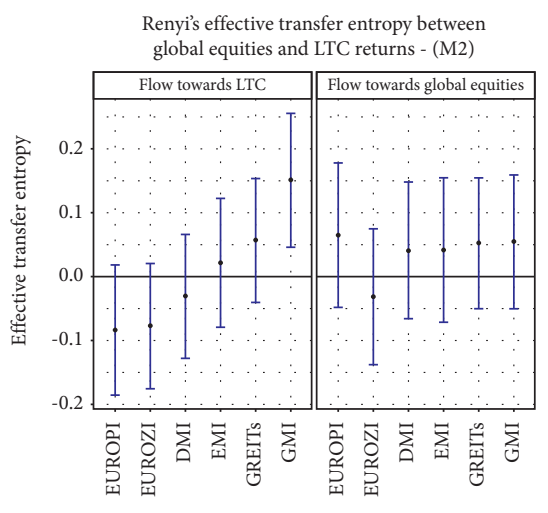

(m)

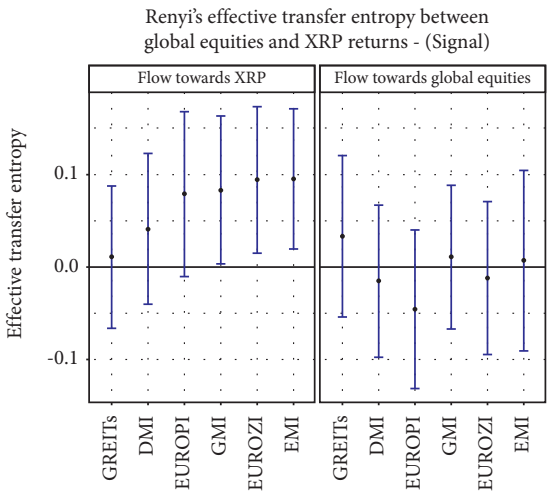

(p)

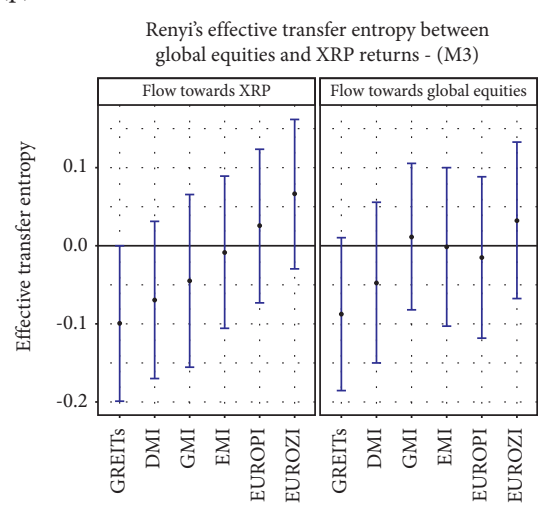

(s)

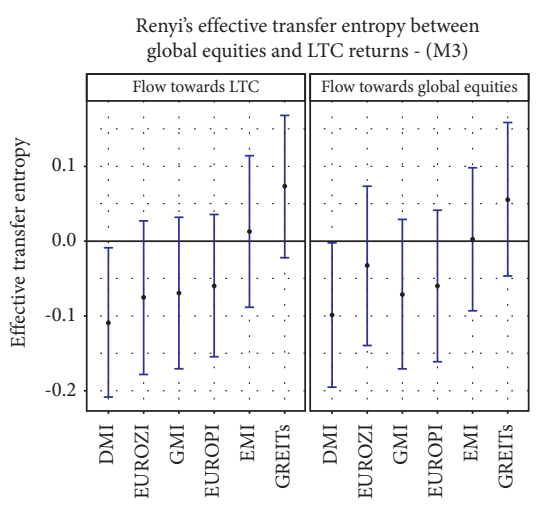

(n)

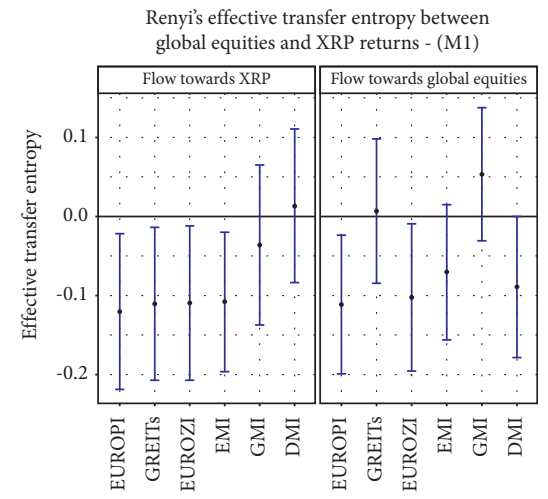

(q)

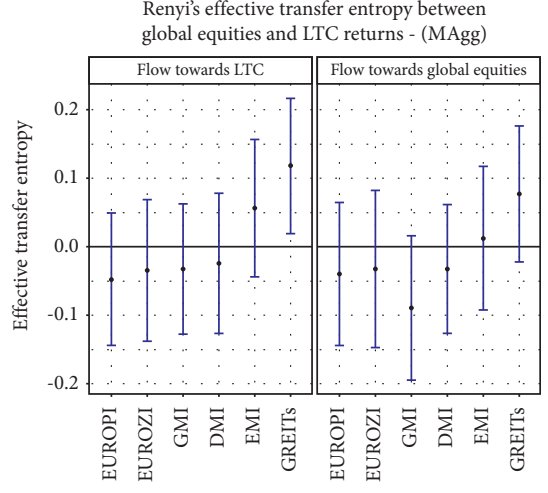

(o)

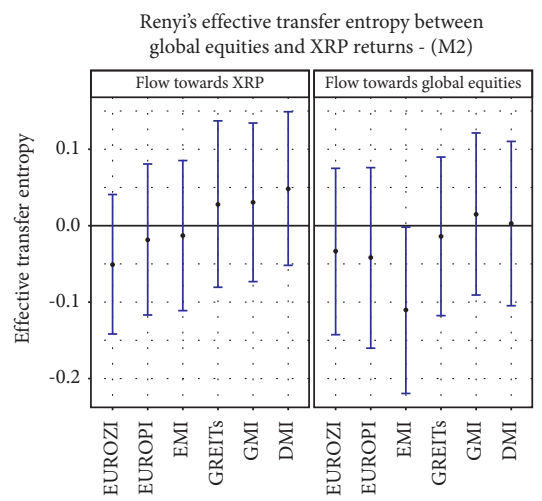

$(\mathrm{r})$

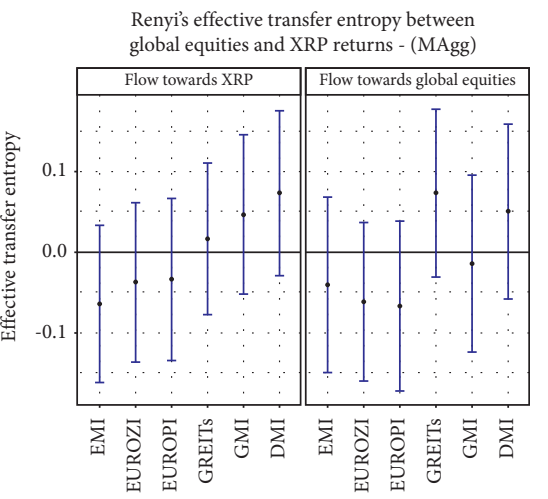

$(\mathrm{t})$

FIGURE 3: Information flow between global equities and cryptocurrencies (pre-COVID-19 pandemic period).

reflect the inefficiency of the Ripple [53]. That is, the patterns of information flow persist from scales M1 to MAgg.

4.2.3. COVID-19 Pandemic Period. The importance of the COVID-19 pandemic period analysis clarifies the patterns of more negative flows from global equities to cryptocurrencies and more positive flows from most cryptocurrencies to global equities. The positive flow from most cryptocurrencies to global equities is not surprising because most cryptocurrencies have been flaunted to exhibit similar behaviour in terms of information flow [90].

From Figure 4, both the original series and multiscales demonstrate potential negative information flows from global equities to Bitcoin. However, the multiscales reveal a significant positive flow from Bitcoin to global equities as compared to the original series. The flow during the COVID19 pandemic may have significant portfolio selection and management benefits. That is, the negative flows from global equities signify that in periods of stress in the market, Bitcoin can act as a safe haven for global equities; the reverse is true from the medium- and long-run perspective for most global equities, except EUROPI, EUROZI, and GMI in the mediumterm. Thus, in the short- to medium-terms, there are positive causal relationships between Bitcoin and the aforementioned equities which minimises diversification, hedges, and safe haven benefits. Notwithstanding, this study contributes to the argument on the existence of a "bubble" in the cryptocurrency market [91, 92]. In addition, the study by Moosa [93] 


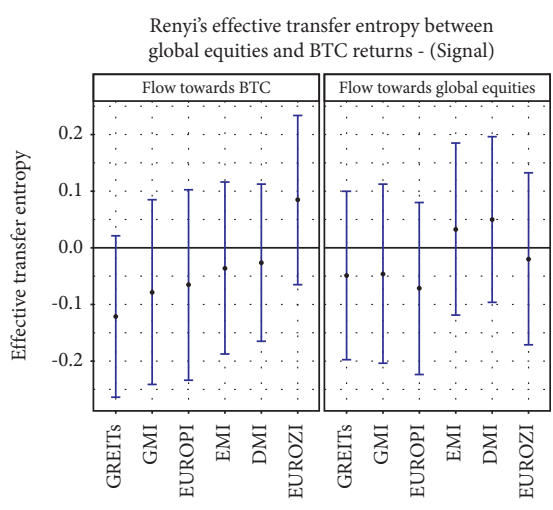

(a)

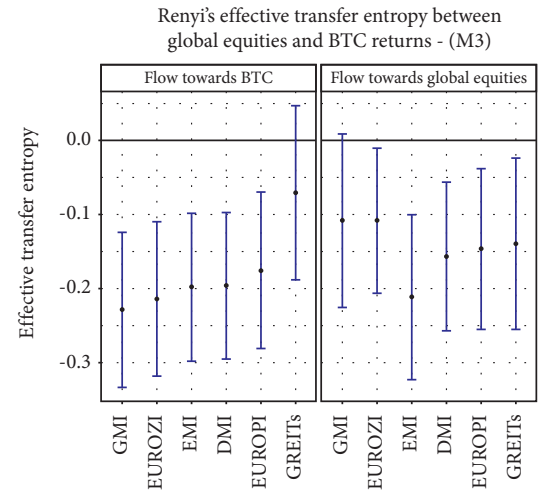

(d)

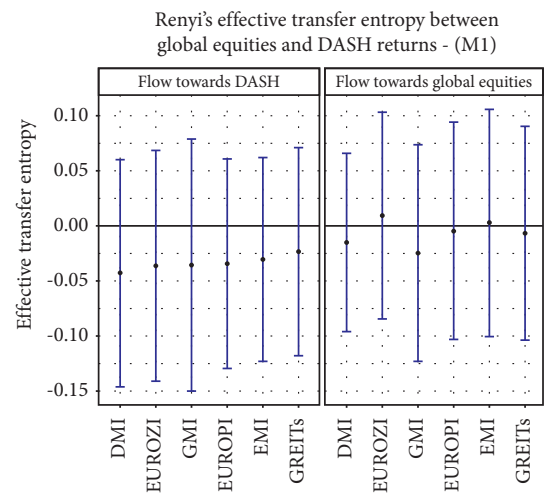

(g)

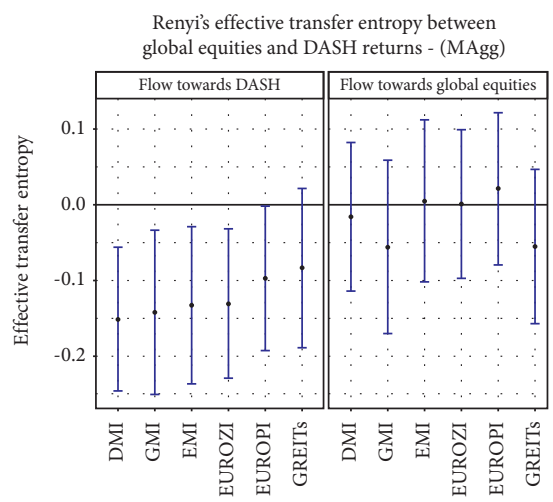

(j)

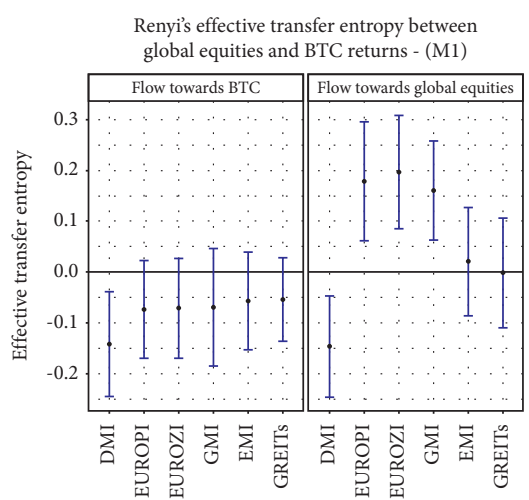

(b)

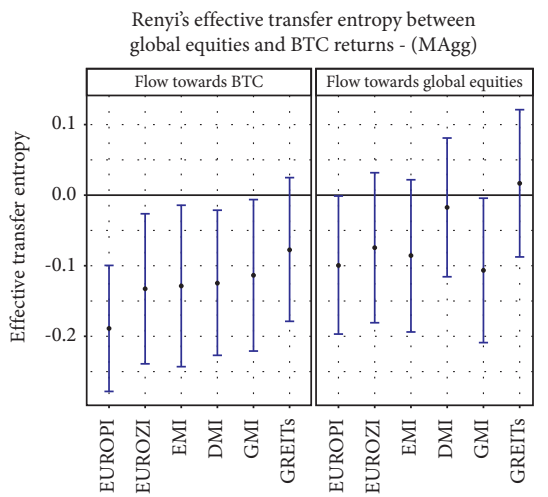

(e)

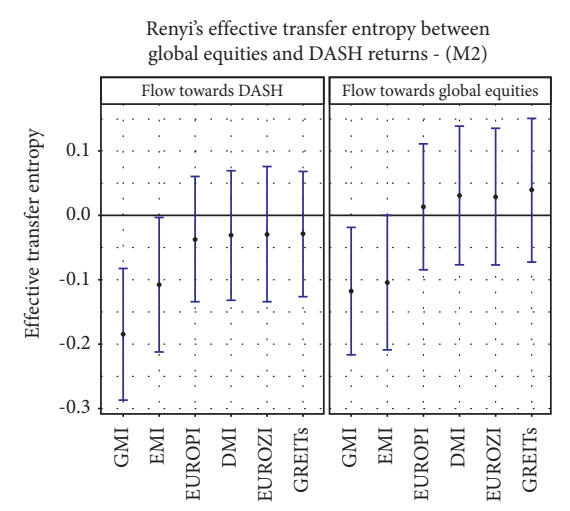

(h)

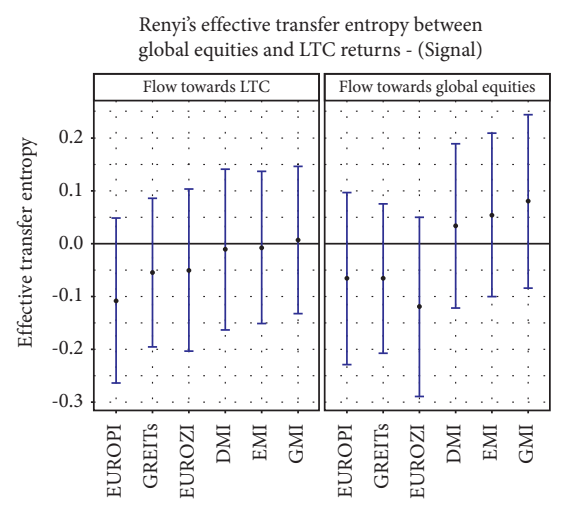

(k)

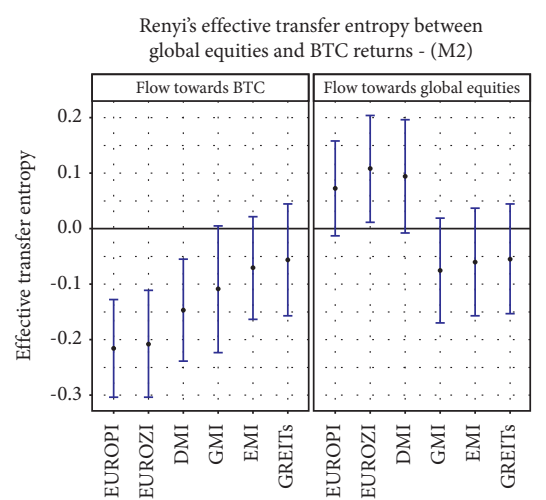

(c)

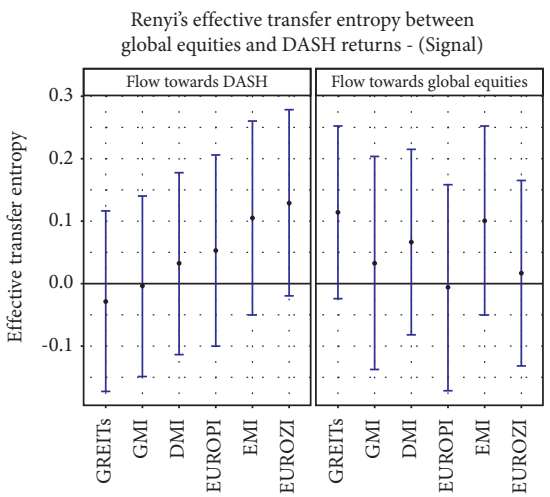

(f)

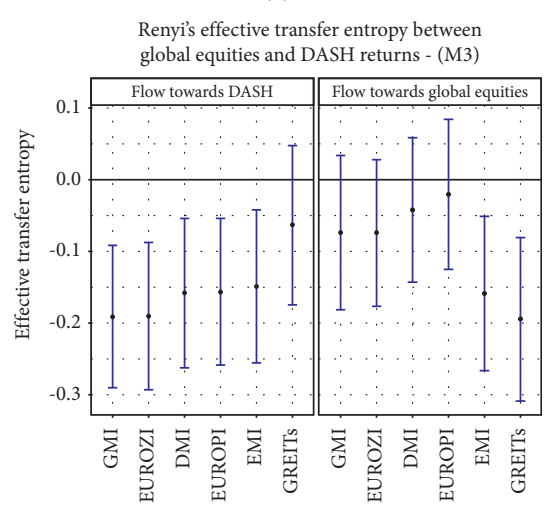

(i)

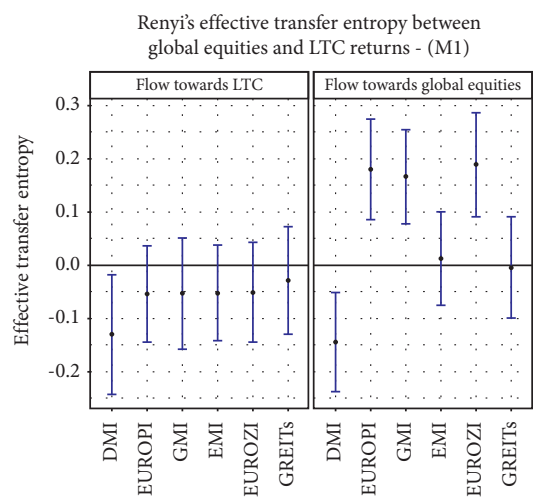

(1)

Figure 4: Continued. 


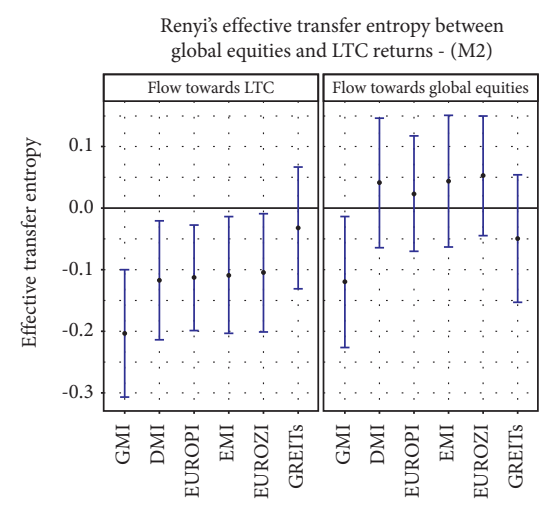

(m)

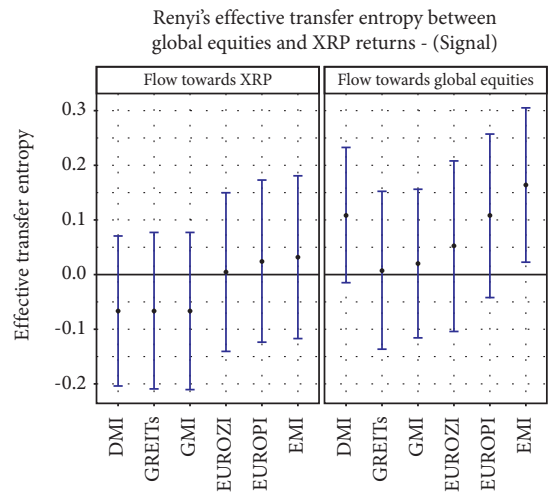

(p)

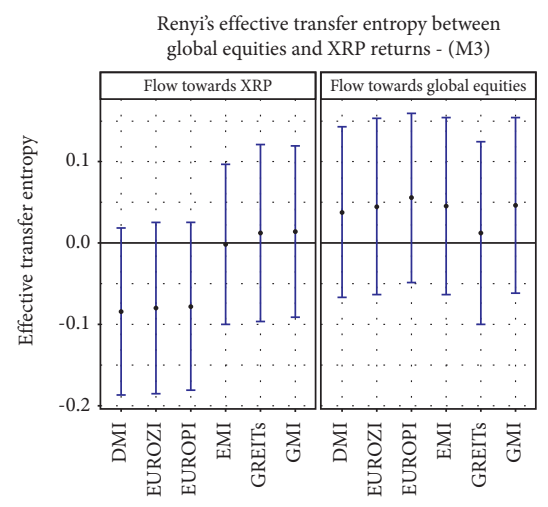

(s)

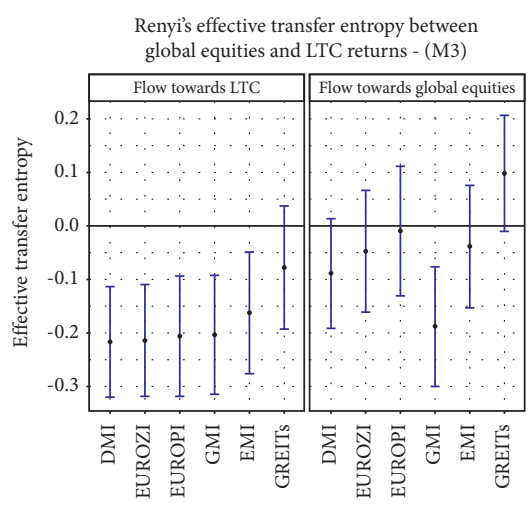

(n)

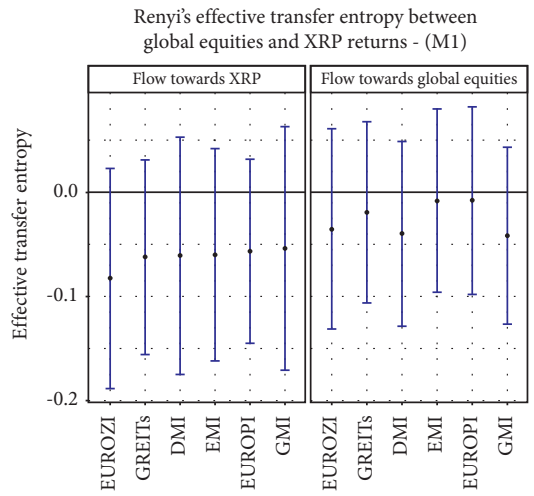

(q)

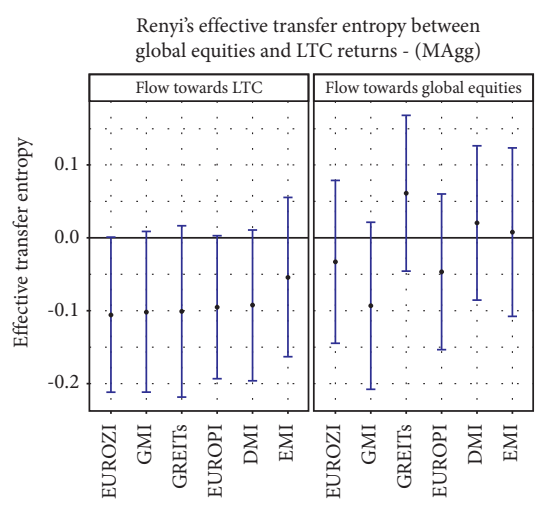

(o)

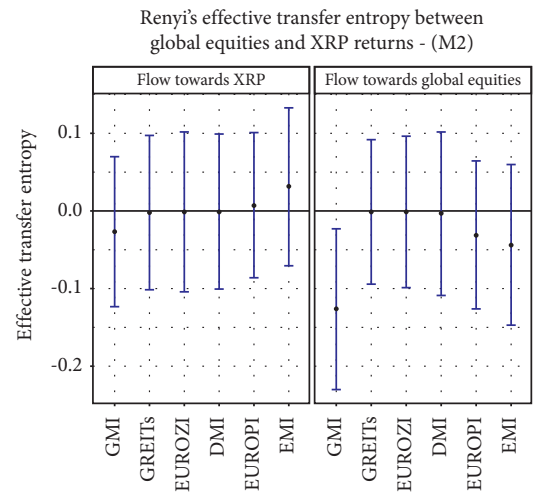

(r)

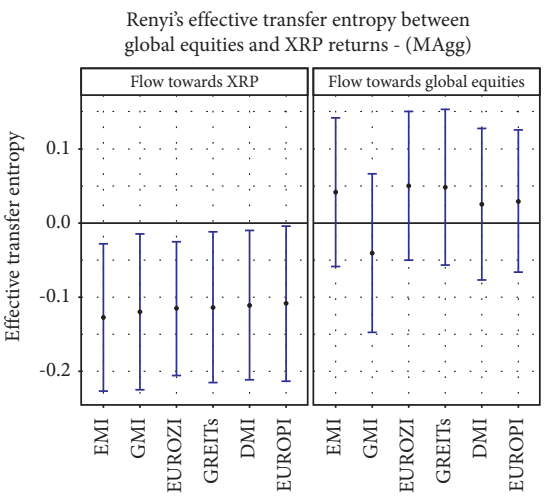

(t)

Figure 4: Information flow between global equities and cryptocurrencies (COVID-19 pandemic period).

postulates that Bitcoin was in a bubble up to the end of 2017. The current study asserts that the volume of trading in Bitcoin and the global equities can be explained predominantly in terms of price dynamics considering past price movements. Specifically, the dynamics of the study depict negative price fluctuations, and that the path of the price is well delineated by financial Contagion [16].

From Figure 4, there seems to be a long memory in the flow of information between global equities and Dash. This establishes strong market inefficiencies in both markets and can be traced from the persistency in the flow of information from scales M1 to MAgg (short-, medium-, and long-terms). This denotes that at extreme market conditions, Dash and global equities can act as a safe haven for each other, following from Baur and Lucey [58].

Moreover, there are more negative flows from global equities to Litecoin analogous to the more positive flow from Litecoin. This clearly shows that in times of market stress, Litecoin serves as a safe haven for global equities but the reverse is not true. Specifically, in the medium- and longterms, there is a negative significant flow of information from global equities to Litecoin.

To end with, from Figure 4, there are more negative significant flows from global equities to Ripple analogous to the more positive flow from Ripple but not significant. This clearly shows that in periods of market stress, Ripple serves as a safe haven for global equities, but the reverse is not true. 


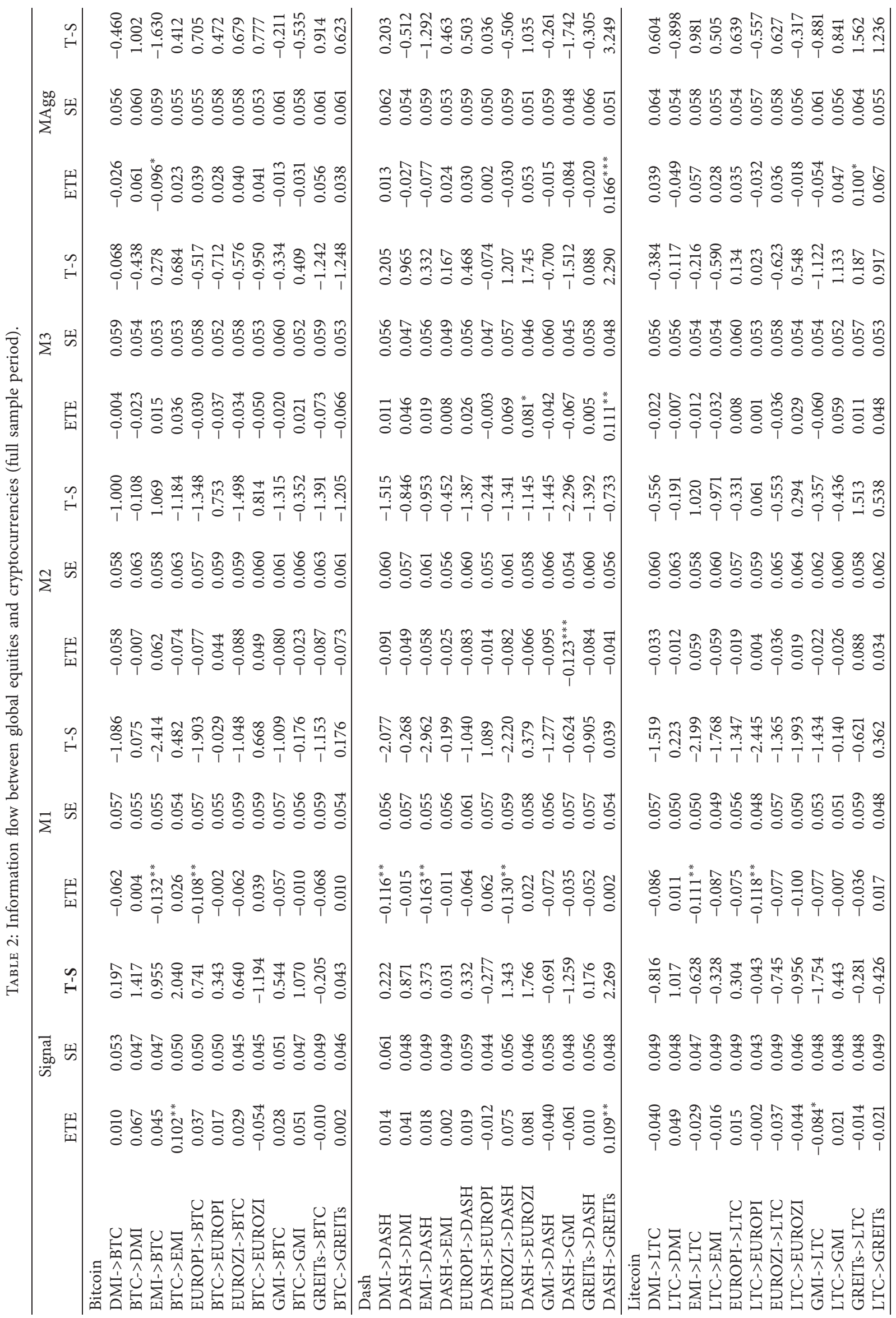




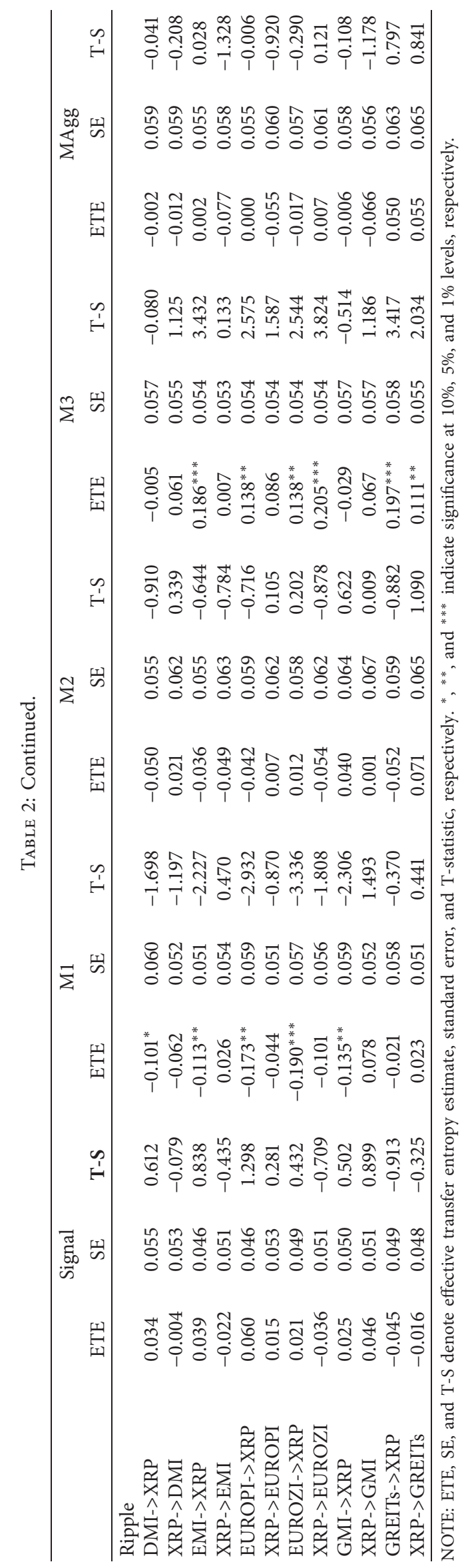




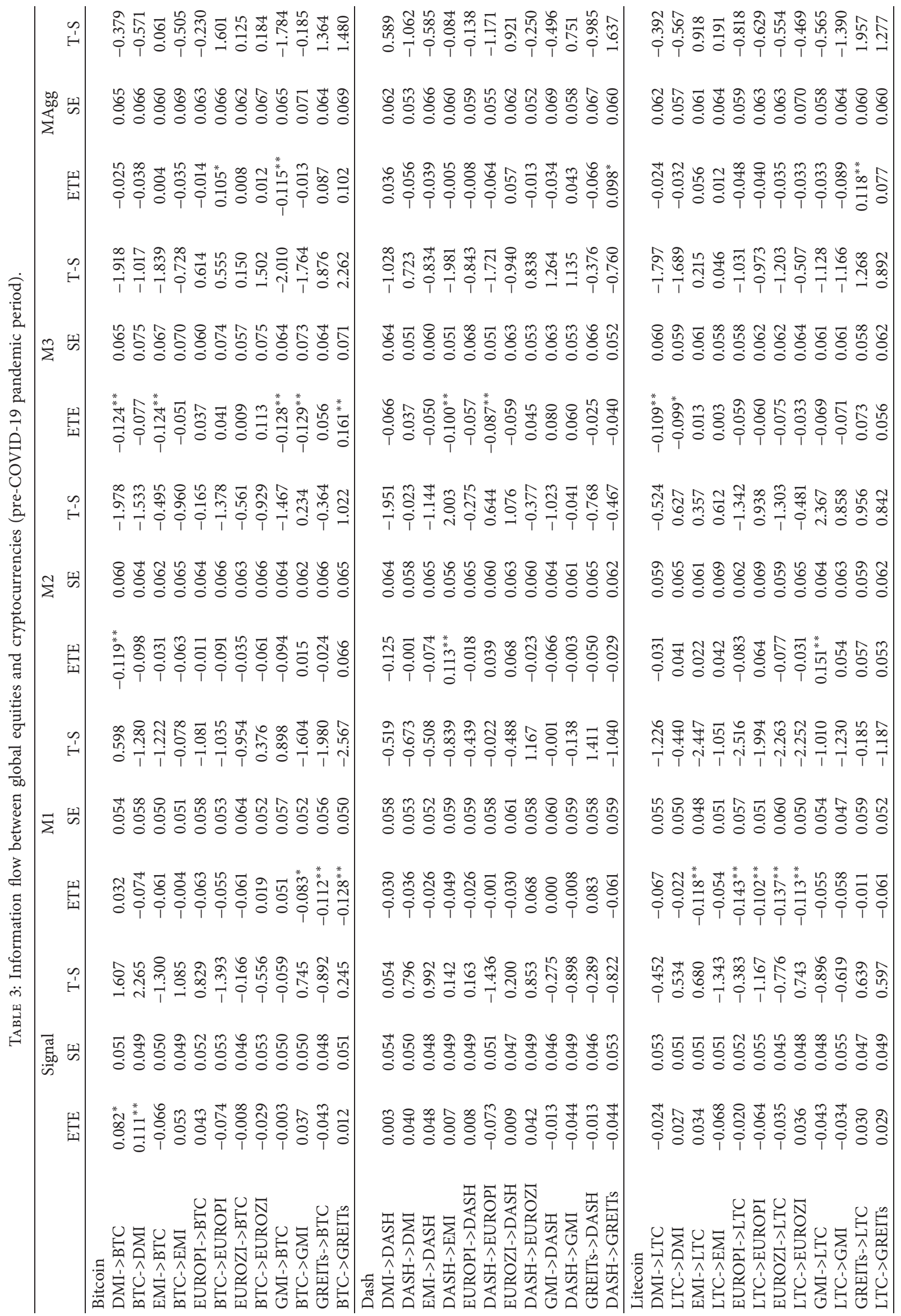




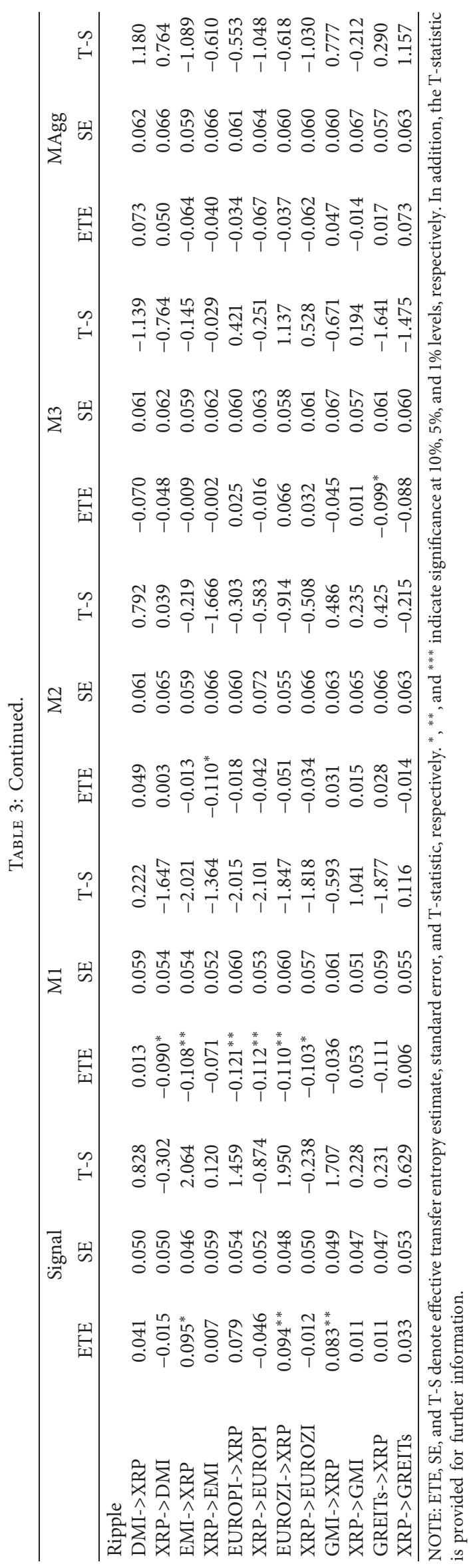




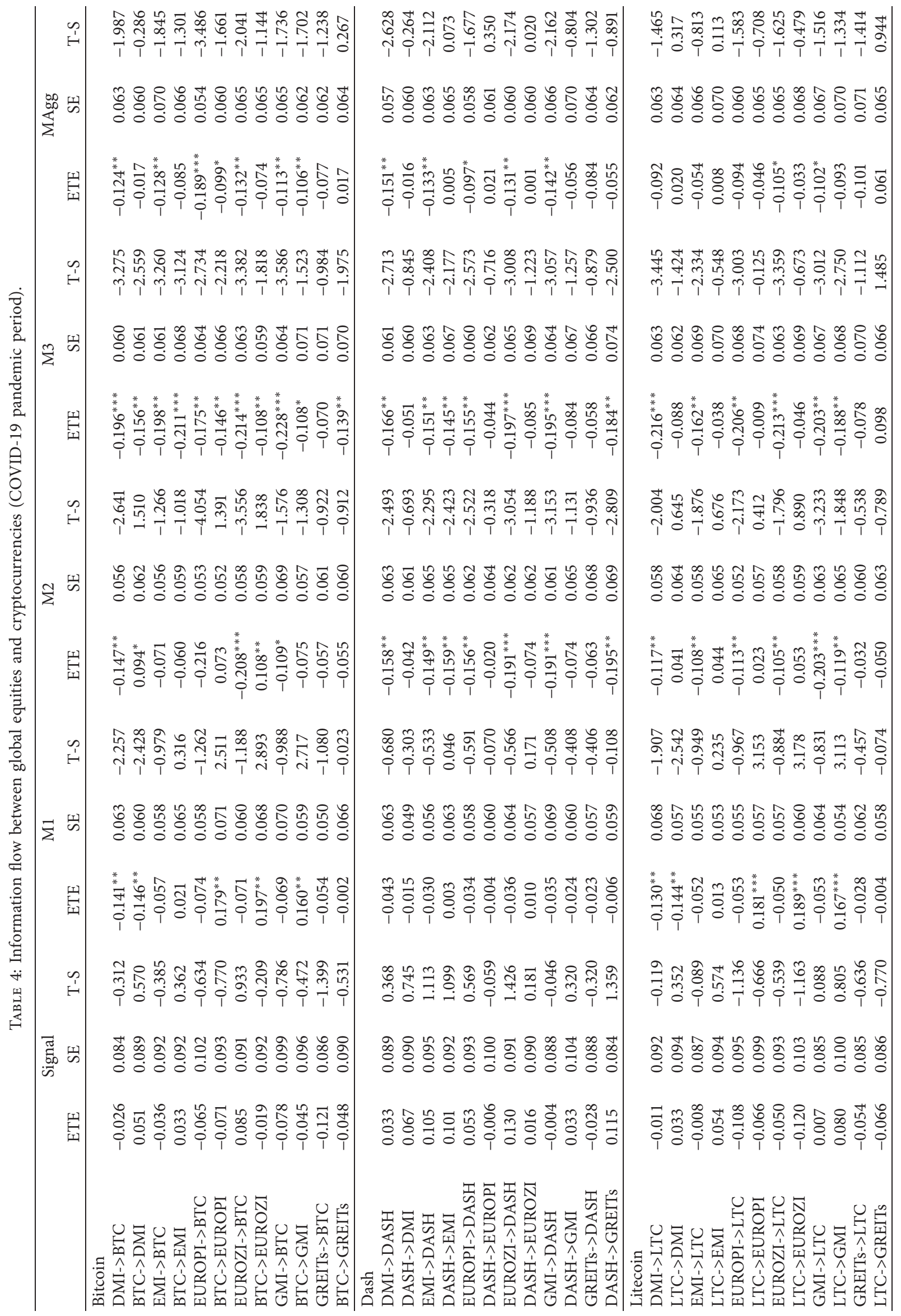




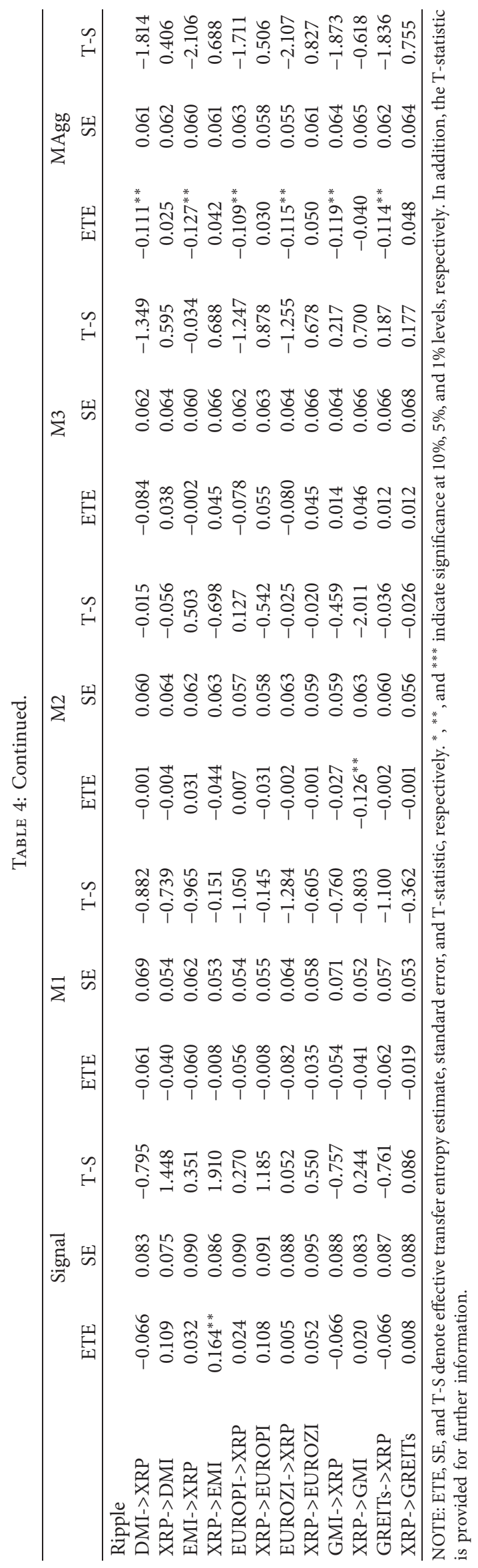


Specifically, in the long-term, there is a negative significant flow of information from global equities. This shows that a portfolio with any of the global equities and Ripple with long-term holdings has Ripple providing safe haven for global equities under extreme market conditions [58].

\section{Conclusion}

We employed the variational mode decomposition-based entropy to quantify the direction and strength of information transfer between global equities and cryptocurrencies at diverse time scales. In this way, we explored the multiscale information that might be ignored while solving the mode mixing problem in the decomposition result by the shift from sifting process via multipliers approach. Due to the nonlinearity of most financial time series, we adopt a loglikelihood ratio transfer entropy which quantifies information from a probability density function. We set $q$ from the Rényi transfer entropy to 0.3 to account for tail events within the sampled time series, which establishes the point that it is extreme events rather than observations in the centre that comes to light when information flow is utilised $[8,81]$.

The fluctuation in information flow from the global equities harmonises the notion of Rua and Nunes [94] and at diverse scales representing the natural rule of scale-invariance of a self-similar process to support the heterogeneous market hypothesis [38]. Most cryptocurrencies demonstrate a similar transmission of information flow [90] with the global equities for each of the sample periods. The COVID19 pandemic period appears to be driving the estimates for the full sample period, which is, a negative flow. Thus, the direction and significance of the information flow between the markets for the full sample correspond to the one observed at the COVID-19 pandemic period. That is, on average, the significant negative information flow to most cryptocurrencies vis-à-vis the insignificant negative flow to global equities observed in the full sample is also evident in the COVID-19 period. It goes to reason that major events from the global equities incite reaction effects and information chutes which lead to a significant downturn of markets. This is apparent from the transfer entropy that knowing the history of cryptocurrencies, in this case, indicates considerably more uncertainty than knowing the history of only global equities. Again, the negative flow from cryptocurrencies adds to the debate on the existence of a "bubble" in the market of cryptocurrencies [91, 92]. However, the negative causal relationships are established by extant literature to offer greater portfolio management benefits [10, 58].

Also, the significant flow of information between global equities and cryptocurrencies establishes the information flow theory, where two random variables are related in such a way that one variable can learn about the state of the other through observation of the other, and then they have mutual information [56]. Correspondingly, positive flows from some global equities concur with the assertion made by $\mathrm{Ji}$ et al. [60] that an increase in trading volumes of cryptocurrencies attracts investors to the cryptocurrencies market due to higher expectations of price stability and minimal uncertainty. Conversely, the negative information flow from cryptocurrencies to some global equities confirms the equity parity theory which may lead to domestic investors repatriating part of their foreign holdings on cryptocurrencies as a reaction to adverse movements in domestic currencies due to the high performance of the foreign or international holdings. That is, as individuals invest in cryptocurrencies as international holdings, domestic equity holdings, which are reflected in the global equity indices, are exposed to adverse exchange rates shocks. It has also become apparent that all market participants display interconnectedness and similarities, which constitute a significant market force.

Furthermore, there have been constant debates on whether cryptocurrencies act as a speculative bubble that could crash or support money laundering. Corollary to this, Giudici et al. ([35], p. 13) argue that "they meet a market need for a faster and more secure payment and transaction system, disintermediating monopolies, banks, and credit cards." On the other hand, critics indicate that the unsteady value of cryptocurrencies makes them more preferred for a speculative asset than a new type of money [15, 16, 91, 92]. The current study advocates that the patterns of causality between cryptocurrencies and global equities vary across investment horizons. Again, the benefits of diversification, hedges and safe haven may be observed at different market conditions. This was revealed by the pre-COVID-19 pandemic (diversification and hedge) as well as the COVID-19 pandemic periods (safe haven). Cryptocurrencies experience unstable dynamics but yet can perform some useful functions such as adding economic value. We strongly support the idea of Giudici, et al. [35] that the inclination is towards the regulation of cryptocurrencies. This is an indispensable step to protect market participants with diverse investment horizons, though this may not augur well for the original libertarian rationale that invented Bitcoin. Accordingly, this may minimise moral hazard and information asymmetries in cryptocurrencies and global equities markets.

Generally, the findings present pertinent inferences for portfolio diversification, policy decisions, investing risk, and risk management schemes in cryptocurrencies and global equities. For the sake of asset allocation and risk management, the support is incumbent on the negative information flow between cryptocurrencies and global equities [88] at various investment horizons. The differences in information flow over diverse investment horizons during the COVID19 provide evidence of contagion [95]. As a result, we advocate that cryptocurrencies are not detached from the financial system but as an effective asset for portfolio analysis with equities $[88,96]$ and clearly act as complementary assets rather than substitutes.

From the aforesaid, future studies can concentrate on country-specific equities whose global equity index recorded significant information flow with cryptocurrencies. Again, these developed relationships can be assessed using a more advanced decomposition technique(s), since multiscales are pertinent in these markets [51]. Further studies can consider portfolio analysis through the various investment horizons for other financial assets at high-, medium-, and low 
frequencies. Also, other studies can employ quantile regression to reveal additional information at various quantiles [97].

\section{Data Availability}

The stock indices data used to support the findings of this study supplied by EquityRT are under license and so cannot be made freely available. Data on cryptocurrencies were obtained from Yahoo Finance and can be made freely available.

\section{Conflicts of Interest}

The authors declare that they have no conflicts of interest.

\section{References}

[1] R. M. Gyasi, "COVID-19 and mental health of older Africans: an urgency for public health policy and response strategy," International Psychogeriatrics, vol. 32, no. 10, pp. 1187-1192, 2020.

[2] S. K. Agyei, Z. Isshaq, S. Frimpong, A. M. Adam, A. Bossman, and O. Asiamah, "COVID-19 and food prices in sub-Saharan Africa," African Development Review, vol. 33, no. 13, pp. 1-12, 2021.

[3] N. Iqbal, Z. Fareed, F. Shahzad, X. He, U. Shahzad, and M. Lina, "The nexus between COVID-19, temperature and exchange rate in Wuhan city: new findings from partial and multiple wavelet coherence," The Science of the Total Environment, vol. 729, Article ID 138916, 2020.

[4] J. W. Goodell and S. Goutte, "Co-movement of COVID-19 and Bitcoin: evidence from wavelet coherence analysis," Finance Research Letters, vol. 38, Article ID 101625, 2021.

[5] S. Lahmiri and S. Bekiros, "The impact of COVID-19 pandemic upon stability and sequential irregularity of equity and cryptocurrency markets," Chaos, Solitons \& Fractals, vol. 138, Article ID 109936, 2020.

[6] J. Wang, W. Shao, and J. Kim, "Analysis of the impact of COVID-19 on the correlations between crude oil and agricultural futures," Chaos, Solitons \& Fractals, vol. 136, Article ID 109896, 2020.

[7] S. Lahmiri and S. Bekiros, "Randomness, informational entropy, and volatility interdependencies among the major world markets: the role of the COVID-19 pandemic," Entropy, vol. 22 , no. 8 , p. 833, 2020.

[8] S. Lahmiri and S. Bekiros, "Renyi entropy and mutual information measurement of market expectations and investor fear during the COVID-19 pandemic," Chaos, Solitons \& Fractals, vol. 139, Article ID 110084, 2020.

[9] D. Chaum, "Blind signatures for untraceable payments," in Advances in Cryptology, pp. 199-203, Springer, Boston, MA, USA, 1983.

[10] P. Owusu Junior, A. M. Adam, and G. Tweneboah, "Connectedness of cryptocurrencies and gold returns: evidence from frequency-dependent quantile regressions," Cogent Economics \& Finance, vol. 8, no. 1, Article ID 1804037, 2020.

[11] S. Nakamoto and A. Bitcoin, "A peer-to-peer electronic cash system,” 2008, https://bitcoin.org/bitcoin.pdf.

[12] Á. Cebrián-Hernández and E. Jiménez-Rodríguez, "Modeling of the bitcoin volatility through key financial environment variables: an application of conditional correlation MGARCH models," Mathematics, vol. 9, no. 3, p. 267, 2021.
[13] T. Papadimitriou, P. Gogas, and F. Gkatzoglou, "The evolution of the cryptocurrencies market: a complex networks approach," Journal of Computational and Applied Mathematics, vol. 376, Article ID 112831, 2020.

[14] A. Brauneis and R. Mestel, "Price discovery of cryptocurrencies: bitcoin and beyond," Economics Letters, vol. 165, pp. 58-61, 2018.

[15] L. A. Gil-Alana, E. J. A. Abakah, and M. F. R. Rojo, "Cryptocurrencies and stock market indices. Are they related?" Research in International Business and Finance, vol. 51, Article ID 101063, 2020.

[16] L. Yarovaya, J. Brzeszczyński, and C. K. M. Lau, "Intra-and inter-regional return and volatility spillovers across emerging and developed markets: evidence from stock indices and stock index futures," International Review of Financial Analysis, vol. 43, pp. 96-114, 2016.

[17] E. F. Fama and K. R. French, Multifactor Explanations of Asset Pricing Anomalies, University of Chicago Press, Chicago, IL, USA, 2021.

[18] A. K. Tiwari, I. D. Raheem, and S. H. Kang, "Time-varying dynamic conditional correlation between stock and cryptocurrency markets using the copula-ADCC-EGARCH model," Physica A: Statistical Mechanics and Its Applications, vol. 535, Article ID 122295, 2019.

[19] S. A. Ross, "The arbitrage theory of capital asset pricing," Journal of Economic Theory, vol. 13, pp. 11-30, 1976.

[20] A. M. Adam and G. Tweneboah, "Macroeconomic factors and stock market movement: evidence from Ghana," SSRN Electronic Journal, pp. 1-19, 2008.

[21] M. Z. Rehman, "The macroeconomic and institutional drivers of stock market development: empirical evidence from BRICS economies," The Journal of Asian Finance, Economics, and Business, vol. 8, no. 2, pp. 77-88, 2021.

[22] P. Iliev, "The effect of SOX section 404: costs, earnings quality, and stock prices," The Journal of Finance, vol. 65, no. 3, pp. 1163-1196, 2010.

[23] Y. Gorodnichenko and M. Weber, "Are sticky prices costly? evidence from the stock market," The American Economic Review, vol. 106, no. 1, pp. 165-199, 2016.

[24] P. C. Tetlock, "Giving content to investor sentiment: the role of media in the stock market," The Journal of Finance, vol. 62, no. 3, pp. 1139-1168, 2007.

[25] R. P. Robins and G. P. Smith, "On unmodeled breaks in the turn of the year, turn of the month, and january effects," Financial Review, vol. 52, no. 4, pp. 725-747, 2017.

[26] E. Bouri, M. Das, R. Gupta, and D. Roubaud, "Spillovers between Bitcoin and other assets during bear and bull markets," Applied Economics, vol. 50, no. 55, pp. 5935-5949, 2018.

[27] E. Asafo-Adjei, A. M. Adam, and P. Darkwa, "Can crude oil price returns drive stock market returns of oil producing countries in Africa? evidence from bivariate and multiple wavelet," Macroeconomics and Finance in Emerging Markets Economies, 2021.

[28] K. C. Shadlen, "Exchanging development for market access? deep integration and industrial policy under multilateral and regional-bilateral trade agreements," Review of International Political Economy, vol. 12, no. 5, pp. 750-775, 2005.

[29] S. M. Bartram and G. Dufey, "International portfolio investment: theory, evidence, and institutional framework," Financial Markets, Institutions \& Instruments, vol. 10, no. 3, pp. 85-155, 2001.

[30] M. E. H. Arouri and P. Foulquier, "Financial market integration: theory and empirical results," Economic Modelling, vol. 29, no. 2, pp. 382-394, 2012. 
[31] P. Owusu Junior, I. Alagidede, and G. Tweneboah, "Shapeshift contagion in emerging markets equities: evidence from frequency-and time-domain analysis," Economics and Business Letters, vol. 9, no. 3, pp. 146-156, 2020.

[32] S. M. F. G. Cavaglia, J. Diermeier, V. Moroz, and S. De Zordo, "Investing in global equities," Journal of Portfolio Management, vol. 30, no. 3, pp. 88-94, 2004.

[33] P. Owusu Junior, S. Frimpong, A. M. Adam et al., "COVID-19 as information transmitter to global equity markets: evidence from CEEMDAN-based transfer entropy approach," Mathematical Problems in Engineering, vol. 2021, Article ID 8258778, 19 pages, 2021.

[34] T. Conlon, S. Corbet, and R. J. McGee, “Are cryptocurrencies a safe haven for equity markets? an international perspective from the COVID-19 pandemic," Research in International Business and Finance, vol. 54, Article ID 101248, 2020.

[35] G. Giudici, A. Milne, and D. Vinogradov, "Cryptocurrencies: market analysis and perspectives," Journal of Industrial and Business Economics, vol. 47, no. 1, pp. 1-18, 2020.

[36] G. Peters, E. Panayi, and A. Chapelle, "Trends in cryptocurrencies and blockchain technologies: a monetary theory and regulation perspective," Journal of Financial Perspectives, vol. 3, no. 3, 2015.

[37] H. Markowitz, "Portfolio selection," The Journal of Finance, vol. 7, no. 1, pp. 77-91, 1952.

[38] U. A. Müller, M. M. Dacorogna, R. D. Davé, R. B. Olsen, O. V. Pictet, and J. E. Von Weizsäcker, "Volatilities of different time resolutions-analyzing the dynamics of market components," Journal of Empirical Finance, vol. 4, no. 2-3, pp. 213-239, 1997.

[39] J. Lian, Z. Liu, H. Wang, and X. Dong, “Adaptive variational mode decomposition method for signal processing based on mode characteristic," Mechanical Systems and Signal Processing, vol. 107, pp. 53-77, 2018.

[40] A. W. Lo, "The adaptive markets hypothesis," Journal of Portfolio Management, vol. 30, no. 5, pp. 15-29, 2004.

[41] K. Dragomiretskiy and D. Zosso, "Variational mode decomposition," IEEE Transactions on Signal Processing, vol. 62, no. 3, pp. 531-544, 2013.

[42] N. E. Huang, Z. Shen, S. R. Long et al., "The empirical mode decomposition and the Hilbert spectrum for nonlinear and non-stationary time series analysis," Proceedings of the Royal Society of London Series A: Mathematical, Physical and Engineering Sciences, vol. 454, no. 1971, pp. 903-995, 1998.

[43] Z. Wu and N. E. Huang, "Ensemble empirical mode decomposition: a noise-assisted data analysis method," Advances in Adaptive Data Analysis, vol. 1, no. 1, pp. 1-41, 2009.

[44] S. Mohanty, K. K. Gupta, and K. S. Raju, "Comparative study between VMD and EMD in bearing fault diagnosis," in Proceedings of the 2014 9th International Conference on Industrial and Information Systems (ICIIS), pp. 1-6, IEEE, Gwalior, India, December 2014.

[45] Z. Li, Y. Jiang, Q. Guo, C. Hu, and Z. Peng, "Multi-dimensional variational mode decomposition for bearing-crack detection in wind turbines with large driving-speed variations," Renewable Energy, vol. 116, pp. 55-73, 2018.

[46] M. F. Isham, M. S. Leong, M. H. Lim, and Z. A. Ahmad, "Variational mode decomposition: mode determination method for rotating machinery diagnosis," Journal of Vibroengineering, vol. 20, no. 7, pp. 2604-2621, 2018.

[47] Z. Wu and N. E. Huang, "Statistical significance test of intrinsic mode functions," in Hilbert-huang Transform and its Applications, pp. 107-127, World Scientific, USA, 2005.
[48] P. M. Osei and A. M. Adam, "Quantifying the information flow between Ghana stock market index and its constituents using transfer entropy," Mathematical Problems in Engineering, vol. 2020, Article ID 6183421, 2020.

[49] A. M. Adam, "Susceptibility of stock market returns to international economic policy: evidence from effective transfer entropy of Africa with the implication for open innovation," Journal of Open Innovation: Technology, Market, and Complexity, vol. 6, no. 3, p. 71, 2020.

[50] X. Su and Y. Li, "Dynamic sentiment spillovers among crude oil, gold, and Bitcoin markets: evidence from time and frequency domain analyses," PLoS One, vol. 15, no. 12, Article ID e0242515, 2020.

[51] W. Kristjanpoller, E. Bouri, and T. Takaishi, "Cryptocurrencies and equity funds: evidence from an asymmetric multifractal analysis," Physica A: Statistical Mechanics and Its Applications, vol. 545, Article ID 123711, 2020.

[52] M. Omane-Adjepong, P. Alagidede, and N. K. Akosah, "Wavelet time-scale persistence analysis of cryptocurrency market returns and volatility," Physica A: Statistical Mechanics and Its Applications, vol. 514, pp. 105-120, 2019.

[53] V. L. Tran and T. Leirvik, "Efficiency in the markets of cryptocurrencies," Finance Research Letters, vol. 35, Article ID 101382, 2020.

[54] E. Bouri, X. V. Vo, and T. Saeed, "Return equicorrelation in the cryptocurrency market: analysis and determinants," Finance Research Letters, vol. 38, Article ID 101497, 2021.

[55] R. Caferra and D. Vidal-Tomás, "Who raised from the abyss? A comparison between cryptocurrency and stock market dynamics during the COVID-19 pandemic," Finance Research Letters, Article ID 101954, 2021.

[56] S. Benthall, "Situated information flow theory," in Proceedings of the 6th Annual Symposium on Hot Topics in the Science of Security, pp. 1-10, Nashville, TN, USA, April 2019.

[57] J. Wang and X. Wang, "COVID-19 and financial market efficiency: evidence from an entropy-based analysis," Finance Research Letters, vol. 42, Article ID 101888, 2021.

[58] D. G. Baur and B. M. Lucey, "Is gold a hedge or a safe haven? an analysis of stocks, bonds and gold," Financial Review, vol. 45, no. 2, pp. 217-229, 2010.

[59] M. Rubinstein, "Markowitz's "portfolio selection:" a fifty-year retrospective," The Journal of Finance, vol. 57, no. 3, pp. 1041-1045, 2002.

[60] Q. Ji, E. Bouri, C. K. M. Lau, and D. Roubaud, "Dynamic connectedness and integration in cryptocurrency markets," International Review of Financial Analysis, vol. 63, pp. 257272, 2019.

[61] C. Gurdgiev and D. O'Loughlin, "Herding and anchoring in cryptocurrency markets: investor reaction to fear and uncertainty," Journal of Behavioral and Experimental Finance, vol. 25, p. 100271, 2020.

[62] N. Kim, K. Lučivjanská, P. Molnár, and R. Villa, "Google searches and stock market activity: evidence from Norway," Finance Research Letters, vol. 28, pp. 208-220, 2019.

[63] J. M. Fleming, "Domestic financial policies under fixed and under floating exchange rates (Politiques finacierieures interieures avec un systeme de taux de change fixe et avec un systeme de taux de change fluctuant) (Politica financiera interna bajo sistemas de tipos de cambio fijos o de tipos de cambio fluctuantes)," Staff Papers-International Monetary Fund, vol. 9, no. 3, pp. 369-380, 1962.

[64] R. Mundell, International Economics, Mundell International Economics, New York, NY, USA, 1968. 
[65] M. Aftab, R. Ahmad, and I. Ismail, "Examining the uncovered equity parity in the emerging financial markets," Research in International Business and Finance, vol. 45, pp. 233-242, 2018.

[66] J.-W. Cho, J. H. Choi, T. Kim, and W. Kim, "Flight-to-quality and correlation between currency and stock returns," Journal of Banking \& Finance, vol. 62, pp. 191-212, 2016.

[67] E. Bartsch, J. Boivin, S. Fischer, and P. Hildebrand, "Dealing with the next downturn: from unconventional monetary policy to unprecedented policy coordination," Macro and Market Perspectives, vol. 105, pp. 1-16, 2019.

[68] M. Doyle, Internationalism: kant. ways of war and peace, 1997.

[69] S. W. Polachek, "Conflict and trade," Journal of Conflict Resolution, vol. 24, no. 1, pp. 55-78, 1980.

[70] F. I. Dretske, Knowledge and the Flow of Information, MIT Press, Cambridge, MA, USA, 1981.

[71] J. Pearl, "Causal inference in statistics: an overview," Statistics Surveys, vol. 3, pp. 96-146, 2009.

[72] M. Umar, N. T. Hung, S. Chen, A. Iqbal, and K. Jebran, "Are stock markets and cryptocurrencies connected?" Singapore Economic Review, pp. 1-16, 2020.

[73] D. Das and M. Kannadhasan, "Do global factors impact bitcoin prices? Evidence from wavelet approach," Journal of Economic Research, vol. 23, pp. 227-264, 2018.

[74] N. Hamilton and M. Ferry, CRAN package: variational mode decomposition, 2017.

[75] S. Behrendt, T. Dimpfl, F. J. Peter, and D. J. Zimmermann, "RTransferEntropy-quantifying information flow between different time series using effective transfer entropy," Software, vol. 10, p. 100265, 2019.

[76] C. E. Shannon, "A mathematical theory of communication," Bell System Technical Journal, vol. 27, no. 3, pp. 379-423, 1948.

[77] S. Kullback and R. A. Leibler, "On information and sufficiency," The Annals of Mathematical Statistics, vol. 22, no. 1, pp. 79-86, 1951.

[78] A. Renyi, Probability Theory, North-Holland Publishing Co, Amsterdam, Netherlands, 1970.

[79] C. Beck and F. Schögl, Thermodynamics of Chaotic Systems: An Introduction (No. 4), Cambridge University Press, Cambridge, UK, 1995.

[80] R. Marschinski and H. Kantz, "Analysing the information flow between financial time series," The European Physical Journal B, vol. 30, no. 2, pp. 275-281, 2002.

[81] T. Dimpfl and F. J. Peter, "The impact of the financial crisis on transatlantic information flows: an intraday analysis," Journal of International Financial Markets, Institutions and Money, vol. 31, pp. 1-13, 2014.

[82] E. Asafo-Adjei, D. Agyapong, S. K. Agyei, S. Frimpong, R. Djimatey, and A. M. Adam, "Economic policy uncertainty and stock returns of Africa: a wavelet coherence analysis," Discrete Dynamics in Nature and Society, vol. 2020, Article ID 8846507, 2020.

[83] D. Zhang, M. Hu, and Q. Ji, "Financial markets under the global pandemic of COVID-19," Finance Research Letters, vol. 36, p. 101528, 2020.

[84] R. F. Engle, "Autoregressive conditional heteroscedasticity with estimates of the variance of United Kingdom inflation," Econometrica, vol. 50, no. 4, pp. 987-1007, 1982.

[85] R. F. Engle and S. Manganelli, "CAViaR," Journal of Business \& Economic Statistics, vol. 22, no. 4, pp. 367-381, 2004.

[86] X. Liang, "The Liang-Kleeman information flow: theory and applications," Entropy, vol. 15, no. 1, pp. 327-360, 2013.
[87] S. M. Jang, E. Yi, W. C. Kim, and K. Ahn, "Information flow between bitcoin and other investment assets," Entropy, vol. 21, no. 11, p. 1116, 2019.

[88] Y.-J. Zhang, E. Bouri, R. Gupta, and S.-J. Ma, "Risk spillover between bitcoin and conventional financial markets: an expectile-based approach," The North American Journal of Economics and Finance, vol. 55, p. 101296, 2021.

[89] Y. Jiang, H. Nie, and W. Ruan, "Time-varying long-term memory in Bitcoin market," Finance Research Letters, vol. 25, pp. 280-284, 2018.

[90] A. F. Bariviera, L. Zunino, and O. A. Rosso, "An analysis of high-frequency cryptocurrencies prices dynamics using permutation-information-theory quantifiers," Chaos: An Interdisciplinary Journal of Nonlinear Science, vol. 28, no. 7, Article ID 075511, 2018.

[91] E.-T. Cheah and J. Fry, "Speculative bubbles in Bitcoin markets? an empirical investigation into the fundamental value of Bitcoin," Economics Letters, vol. 130, pp. 32-36, 2015.

[92] C. Baek and M. Elbeck, "Bitcoins as an investment or speculative vehicle? a first look," Applied Economics Letters, vol. 22, no. 1, pp. 30-34, 2015.

[93] I. A. Moosa, "The bitcoin: a sparkling bubble or price discovery?" Journal of Industrial and Business Economics, vol. 47, no. 1, pp. 93-113, 2020.

[94] A. Rua and L. C. Nunes, "International comovement of stock market returns: a wavelet analysis," Journal of Empirical Finance, vol. 16, no. 4, pp. 632-639, 2009.

[95] P. O. Junior, G. Tweneboah, and A. M. Adam, "Interdependence of major exchange rates in Ghana: a wavelet coherence analysis," Journal of African Business, vol. 20, no. 3, pp. 407-430, 2019.

[96] E. Bouri, B. Lucey, and D. Roubaud, "Cryptocurrencies and the downside risk in equity investments," Finance Research Letters, vol. 33, Article ID 101211, 2020.

[97] E. Boateng, A. M. Adam, and P. O. Junior, "Modeling the heterogeneous relationship between the crude oil implied volatility index and African stocks in the coronavirus pandemic," Resources Policys, vol. 74, Article ID 102389, 2021. 